\title{
A Near-ML Complex $K$-Best Decoder with Efficient Search Design for MIMO Systems
}

\author{
Chung-Jung Huang, Chih-Sheng Sung, and Ta-Sung Lee \\ Department of Electrical Engineering, National Chiao Tung University, 1001, Ta-Hsueh Road, Hsinchu 300, Taiwan \\ Correspondence should be addressed to Chung-Jung Huang, chesterhuang.cm96g@g2.nctu.edu.tw
}

Received 21 May 2010; Revised 20 October 2010; Accepted 16 December 2010

Academic Editor: Athanasios Rontogiannis

Copyright ( $) 2010$ Chung-Jung Huang et al. This is an open access article distributed under the Creative Commons Attribution License, which permits unrestricted use, distribution, and reproduction in any medium, provided the original work is properly cited.

\begin{abstract}
A low-complexity near-ML $K$-Best sphere decoder is proposed. The development of the proposed $K$-Best sphere decoding algorithm (SDA) involves two stages. First, a new candidate sequence generator (CSG) is proposed. The CSG directly operates in the complex plane and efficiently generates sorted candidate sequences with precise path weights. Using the CSG and an associated parallel comparator, the proposed $K$-Best SDA can avoid performing a large amount of path weight evaluations and sorting. Next, a new search strategy based on a derived cumulative distribution function (cdf), and an associated efficient procedure is proposed. This search procedure can be directly manipulated in the complex plane and performs ML search in a few preceding layers. It is shown that incorporating detection ordering into the proposed SDA offers a systematic method for determining the numbers of required ML search layers. With the above features, the proposed SDA is shown to provide near ML performance with a lower complexity requirement than conventional $K$-Best SDAs.
\end{abstract}

\section{Introduction}

Next-generation wireless communication systems are expected to provide users with higher data rate services for video, audio, data, and voice signals. Many innovative techniques have recently been proposed to improve the spectral efficiency and reliability of wireless communication links. Some popular examples include coded multicarrier modulation, smart antennas, in particular multiple-input multiple-output (MIMO) technology [1-4], and adaptive modulation $[5,6]$.

Various signal detection schemes can be adopted in MIMO systems, such as linear detection, successive interference cancellation (SIC) $[7,8]$, and maximum-likelihood (ML) detection. Linear detection and SIC scheme are both easy to implement, but their detection performance is not optimal. ML detection is the optimal detection scheme, but its complexity grows exponentially with the size of the transmitted symbol alphabet and number of transmit antennas. To reduce the complexity of ML detection, the sphere decoding algorithm (SDA) has been introduced to achieve the same performance as ML detection with reduced complexity [9-12]. The SDA has received considerable attention as an effective detection scheme for MIMO systems.

The basic idea of the SDA is to locate the lattice point nearest to the received signal vector within a given sphere radius. In doing so, the SDA transforms the original problem into a tree search problem. Some candidate enumeration strategies have been proposed [9-12]. The Fincke and Pohst SDA (FP-SDA) $[9,10]$ sets the radius as a scaled variance of the noise. If no lattice points satisfy the radius constraint, the algorithm increases the search radius and restarts the search. The Schnorr and Euchner SDA (SE-SDA) [12] is a variant of the FP-SDA. It shows that enumerating candidate symbols in ascending order based on their distance from the Babai estimate [13] (nulling-canceling solution) speeds up the tree search. This approach is likely to find the optimal solution faster than the FP-SDA and hence can reduce the computational complexity. With these efforts, the conventional SDA is still too complex in the low SNR regime, and its decoding throughput is not stable in general. Hence, it is not desirable for real-time detection and hardware implementation. Previous works [14-16] proposed some architectures to explore the parallelism property of VLSI 
to improve the decoding throughput. These designs exhibit excellent performance in the higher SNR regime.

To overcome the drawbacks of the conventional SDA, the $K$-Best SDA has been introduced in [17-19]. The $K$ Best SDA uses a breadth-first search and keeps the $K$-Best candidates of each layer for the search of the next layer. Briefly, the main idea of the $K$-Best SDA is to keep only $K$ candidates which have the smallest path weights as the most promising solutions. Hence, the decoding throughput of the $K$-Best SDA is stable. Unfortunately, applying a sorting algorithm to find the $K$-Best candidates in each layer requires many computational operations and a long decoding latency. Moreover, the value of $K$ must be large enough to achieve near-ML performance, and this would increase the computational complexity, decoding latency, and implementation cost.

Sorting is a critical factor in reducing the complexity of a $K$-Best SDA. In [17], the bubble sort algorithm is applied to conduct sorting. More efficient sorting algorithms $[18,19]$ have also been adopted to reduce computational complexity. Recently, a high-efficiency sorting architecture has been proposed, which can sort $K$ values of partial Euclidean distances in $K / 2$ clock cycles [20]. It is found that the quicksort algorithm [18] is not always more suitable than the bubble sort algorithm for a small value of $K$. Some efficient early-pruning schemes have been proposed in $[18,21]$, which eliminate the survival candidates that are unlikely to become ML solutions in the early search layers. The approach in [22] reduces the number of candidate nodes by adopting dynamic $K$ values according to the index of search layers. The above approaches can effectively reduce decoding complexity but also introduce performance degradation due to that the ML solution will inevitably be dropped.

To solve the above performance problem, the method presented in [23] always conducts the ML search in several preceding search layers, where ML search refers to an exhaustive search in a certain layer. In this case, the operation in the remaining layers is the same as the conventional $K$ Best SDA. This approach is a special case of the dynamic- $K$ method, and increases complexity and power consumption significantly. In general, it is not necessary to perform the ML search especially when the channel condition is good. The method proposed in [24] chooses the optimal $K$ dynamically according to the channel condition. An approximated algorithm [25] has been proposed to estimate channel conditions in an efficient way. Nevertheless, these methods require complicated procedures and some extra circuits. To the best of our knowledge, there are no efficient mechanisms for deciding the number of layers in which the ML search is conducted, or whether to perform the ML search under different $K$ values and antenna numbers.

Most of the SDAs developed so far work in the real domain using the real-valued decomposition (RVD) $[17,26$, 27]. Although the real-domain approaches lead to better performance and lower complexity, they require more search layers than the complex domain approaches $[28,29]$. To reduce the number of search layers, some novel search methods which operate in the complex plane have been proposed $[30,31]$. These methods introduce errors when evaluating path weights, achieving the goal of reducing complexity but sacrificing performance significantly. On the other hand, some communication systems require rotating the constellation by a predefined angle before transmitting symbols to achieve a higher diversity gain. In this case, conventional real-domain SDAs cannot be adopted directly, and some extra and complicated techniques are needed. To tackle these issues, a new SDA directly performing in the complex domain is desired.

In this paper, we first propose a simple and efficient complex-domain candidate sequence generator (CSG). The CSG is developed based on the fact that neighboring points share the same candidate sequence in the complex plane, rendering the relevant rule invariant to constellation rotation. With a minor modification, the proposed decoder can be easily applied to wireless communication systems with constellation prerotation to obtain a larger diversity gain. By combining the proposed CSG with an efficient sorting architecture, the proposed decoder can significantly reduce path weight calculations and comparison operations without sacrificing detection performance. Moreover, to address the performance issue, a new search strategy that incorporates the ML search in the preceding layers under poor channel conditions (i.e., channel matrix is ill conditioned) improves the performance of the proposed $K$-Best SDA even when the value of $K$ is small. A judicious criterion is proposed that helps determine fewer ML search layers than previous works $[23,27]$. An efficient search procedure is also proposed that fully utilizes existing hardware elements. The procedure increases hardware utilization and significantly reduces implementation cost. Combining the above features, the proposed $K$-Best SDA exhibits lower complexity, excellent performance, and is well suited to real-time applications.

The remainder of this paper is organized as follows. Section 2 describes the signal model and $K$-Best SDA. Section 3 introduces the proposed candidate generator in the complex plane and its associated sorting algorithm and hardware architecture. Section 4 examines the preprocessing unit, proposes a new search strategy, and presents a comprehensive complexity analysis. Section 5 gives the simulation results to demonstrate the advantages of the proposed SDA. Finally, Section 6 concludes the paper.

Throughout this paper, vectors and matrices are denoted using lower-case and upper-case boldface letters, respectively, with $I_{N}$ representing the $N \times N$ identity matrix. $[\cdot]^{T}$ denotes the transpose operation, and $[\cdot]^{H}$ denotes the conjugate transpose operation. The expectation operator is denoted as $\mathrm{E}[\cdot]$, and $\sim$ means distributed as. $\bmod (\cdot)$ denotes the modulus operation. $\operatorname{Re}(\cdot)$ and $\operatorname{Im}(\cdot)$ are the real and imaginary parts of its argument. $\lceil\cdot\rceil$ and $\lfloor\cdot\rfloor$ denote the ceiling and floor operations, respectively. $\mathbb{Z}, \mathbb{R}$, and $\mathbb{C}$ refer to the field of integer numbers, the field of real numbers, and the field of complex numbers, respectively.

\section{Signal Model and $K$-Best SDA}

Consider an MIMO system with $N$ transmit antennas and $M$ receive antennas. The received signal vector is denoted as 
$\mathbf{y}=\left[\begin{array}{llll}y_{1} & y_{2} & \cdots & y_{M}\end{array}\right]^{T} \in \mathbb{C}^{M \times 1}$, where $y_{m}$ is the received signal at the $m$ th receive antenna. Similarly, the transmitted signal vector is denoted as $\mathbf{x}=\left[\begin{array}{llll}x_{1} & x_{2} & \cdots & x_{N}\end{array}\right]^{T} \in \mathbb{Z}^{N}[j]$, where $\mathbb{Z}[j]:=\{a+j b \mid a, b \in \mathbb{Z}\}$ is the set of Gaussian integers and $x_{n}$ is the transmitted signal at the $n$th transmit antenna. The transmitted signal constellation is assumed to be either 16-QAM or 64-QAM. Assume that $M \geq N$ and that the channel responses are frequency-flat fading and remain constant during a frame transmission. The channel matrix can be expressed as

$$
\mathbf{H}=\left[\begin{array}{cccc}
h_{1,1} & h_{1,2} & \cdots & h_{1, N} \\
h_{2,1} & h_{2,2} & \cdots & h_{2, N} \\
\vdots & \vdots & \ddots & \vdots \\
h_{M, 1} & h_{M, 2} & \cdots & h_{M, N}
\end{array}\right]
$$

where $h_{i, j}$ is the channel gain from the $j$ th transmit antenna to the $i$ th receive antenna. Assuming that there is sufficient antenna separation at the transmit and receive sites, the entries of the channel matrix $\mathbf{H}$ can be regarded as i.i.d. complex Gaussian random variables with zero-mean and unit variance. The relationship between the received signal vector and the transmitted signal vector can be expressed as

$$
\mathbf{y}=\mathbf{H x}+\mathbf{n}
$$

where $\mathbf{n}=\left[\begin{array}{llll}n_{1} & n_{2} & \cdots & n_{M}\end{array}\right]^{T} \in \mathbb{C}^{M \times 1}$ is the i.i.d. complex additive white Gaussian noise (AWGN) vector with zeromean and covariance matrix $\sigma^{2} \mathrm{I}_{M}$.

The optimal detector for MIMO systems is the ML detector, which searches all possible combinations of transmitted symbols via the following criterion [10]:

$$
\widehat{\mathbf{x}}=\arg \min _{\mathbf{x} \in S}\|\mathbf{y}-\mathbf{H} \mathbf{x}\|^{2},
$$

where $S=O^{N}$ denotes the set of all possible transmitted symbol vectors and $O$ is the modulation symbol alphabet set with a size of $M_{c}$. The computational complexity of ML detection grows exponentially with $N$. Therefore, it is difficult to be implemented at the receiver in practice.

The basic idea of the SDA is to restrict the search region of the optimal solution to a smaller subset. Typically, the search region is constrained to the interior of a hypersphere of radius $d$ centered around the received signal $\mathbf{y}$ as described by [10]

$$
d^{2} \geq\|\mathbf{y}-\mathbf{H} \mathbf{x}\|^{2}
$$

First, performing complex QR-decomposition to the channel matrix produces

$$
\mathbf{H}=\left[\begin{array}{ll}
\mathbf{Q}_{1} & \mathbf{Q}_{2}
\end{array}\right]\left[\begin{array}{l}
\mathbf{R} \\
\mathbf{0}
\end{array}\right],
$$

where $\mathbf{Q}_{1} \in \mathbb{C}^{M \times N}$ and $\mathbf{Q}_{2} \in \mathbb{C}^{M \times(M-N)}$ are unitary matrices, $\mathbf{R}$ is an $N \times N$ upper triangular matrix, and $\mathbf{0}$ is an $(M-N) \times N$ zero matrix. Substituting (5) into (4), we have

$$
\left(d^{\prime}\right)^{2} \geq\left\|\mathbf{y}^{\prime}-\mathbf{R} \mathbf{x}\right\|^{2}
$$

where $\mathbf{y}^{\prime}=\mathbf{Q}_{1}^{H} \mathbf{y}$ and $\left(d^{\prime}\right)^{2}=d^{2}-\left\|\mathbf{Q}_{2}^{H} \mathbf{y}\right\|^{2}$. The right-hand side of (6) can be expanded as

$$
\begin{aligned}
\left(d^{\prime}\right)^{2} \geq & \left\|\mathbf{y}^{\prime}-\mathbf{R} \mathbf{x}\right\|^{2}=\sum_{i=1}^{N}\left\|y_{i}^{\prime}-\sum_{j=i}^{N} r_{i, j} x_{j}\right\|^{2} \\
= & \left\|y_{N}^{\prime}-r_{N, N} x_{N}\right\|^{2} \\
& +\left\|y_{N-1}^{\prime}-r_{N-1, N} x_{N}-r_{N-1, N-1} x_{N-1}\right\|^{2}+\cdots \\
= & r_{N, N}^{2}\left\|y_{N}^{\prime \prime}-x_{N}\right\|^{2}+r_{N-1, N-1}^{2}\left\|y_{N-1}^{\prime \prime}-x_{N-1}\right\|^{2}+\cdots,
\end{aligned}
$$

where $y_{i}^{\prime \prime}=\left(y_{i}^{\prime}-\sum_{j=i+1}^{N} r_{i, j} x_{j}\right) / r_{i, i}$. Define the path weight $P_{k}$ and branch weight $B_{k}$ of the $k$ th layer as

$$
\begin{gathered}
P_{k}=0, \quad \text { for } k=N+1 \\
P_{k}=P_{k+1}+B_{k}, \quad \text { for } 1 \leq k \leq N, \\
B_{k}=r_{k, k}^{2}\left\|y_{k}^{\prime \prime}-x_{k}\right\|^{2} .
\end{gathered}
$$

The path weight $P_{k}$ is the partial Euclidean distance (PED) which is a positive and nondecreasing function of $k$. The iterative search for the candidates $x_{N}, x_{N-1}, \ldots, x_{2}, x_{1}$ can be easily transformed into a tree search problem [10]. The decoding process of the $K$-Best SDA can then be regarded as descending a tree in which each parent node has $M_{c}$ branches.

The main idea of the $K$-Best SDA is to keep only the $K$ candidates with the smallest path weights as the most promising solutions. The procedure of the complex $K$-Best SDA is summarized as Algorithm 1.

In (8), path weights are defined for a given candidate symbol $x$. When performing the decoding procedure of Step 2, multiple candidate symbols need to be evaluated concurrently for finding the optimal solution. Therefore, a multi-index notation is needed, and Step 2 can be further elaborated as follows.

Let $P_{i}^{1}, P_{i}^{2}, \ldots, P_{i}^{K}$ denote the $K$ smallest PEDs in the $i$ th layer, where $P_{i}^{1} \leq P_{i}^{2} \leq \cdots \leq P_{i}^{K}$. In performing search in the $(i-1)$ th layer, first conduct full path expansion from the $K$ parent nodes to obtain $K M_{c}$ branch weights $B_{i-1}^{1,1}, B_{i-2}^{1,2}, \ldots, B_{i-1}^{1, M_{c}}, \ldots, B_{i-1}^{K, 1}, B_{i-2}^{K, 2}, \ldots, B_{i-1}^{K, M_{c}}$ and PEDs $P_{i-1}^{1,1}$, $P_{i-2}^{1,2}, \ldots, P_{i-1}^{1, M_{c}}, \ldots, P_{i-1}^{K, 1}, P_{i-2}^{K, 2}, \ldots, P_{i-1}^{K, M_{c}}$, respectively, where $B_{i}^{m, n}$ and $P_{i-1}^{m, n}$ are the branch weight and PED of the $n$th path expanded from the $m$ th parent node. The associated PED of each designated node can be evaluated according to $P_{i-1}^{m, n}=$ $P_{i}^{m}+B_{i-1}^{m, n}$. Next, sort the $K M_{c}$ PEDs, and select $K$ partial nodes having the smallest PEDs among the whole candidate set. The above operations are illustrated in Figure 1. 
Step 1.

(a) Set $k=N$. For each symbol in the complex-plane constellation, calculate $P_{N}=B_{N}$.

(b) Choose those symbols having the $K$ smallest paths.

Step 2.

(a) $k \leftarrow k-1$.

(b) Path Evaluation: For each partial symbol vector that survives the previous

layer; for each symbol in the complex-plane constellation, calculate: $P_{k}=P_{k+1}+B_{k}$.

(c) Sorting and candidate selection: Sort the $K M_{c}$ PEDs, and select $K$ partial

nodes having the smallest PEDs among the entire candidate set.

Step 3.

If $k=1$

Output the vector with the smallest path weight as the estimated solution.

Else

Go back to Step 2.

Algorithm 1

A popular alternative to the complex $K$-Best SDA works in the real domain by performing RVD on the complex signal model

$$
\begin{array}{ll}
\tilde{\mathbf{y}}=\left[\begin{array}{c}
\operatorname{Re}(\mathbf{y}) \\
\operatorname{Im}(\mathbf{y})
\end{array}\right], \quad \tilde{\mathbf{x}}=\left[\begin{array}{c}
\operatorname{Re}(\mathbf{x}) \\
\operatorname{Im}(\mathbf{x})
\end{array}\right], \\
\tilde{\mathbf{n}}=\left[\begin{array}{c}
\operatorname{Re}(\mathbf{n}) \\
\operatorname{Im}(\mathbf{n})
\end{array}\right], \quad \tilde{\mathbf{H}}=\left[\begin{array}{cc}
\operatorname{Re}(\mathbf{H}) & -\operatorname{Im}(\mathbf{H}) \\
\operatorname{Im}(\mathbf{H}) & \operatorname{Re}(\mathbf{H})
\end{array}\right],
\end{array}
$$

which yield

$$
\tilde{\mathbf{y}}=\tilde{\mathbf{H}} \tilde{\mathbf{x}}+\tilde{\mathbf{n}}
$$

where $\tilde{\mathbf{H}} \in \mathbb{R}^{2 M \times 2 N}, \tilde{\mathbf{y}} \in \mathbb{R}^{2 M \times 1}, \tilde{\mathbf{n}} \in \mathbb{R}^{2 M \times 1}$, and $\tilde{\mathbf{x}} \in$ $\Lambda^{2 N \times 1} \subset \mathbb{Z}^{2 N \times 1}$. Note that $\Lambda=\{-3,-1,1,3\}$ for 16 QAM and $\Lambda=\{-7,-5,-3,-1,1,3,5,7\}$ for 64-QAM. After RVD, each component $\tilde{x}_{i}$ of $\widetilde{\mathbf{x}}$ is chosen from a set $\Lambda$ of integer numbers with $\sqrt{M_{c}}$ elements. Since (10) has the same algebraic structure as (2), the complex detection problem can be solved in the real domain using the same $\mathrm{K}$-Best algorithm. This is denoted as the conventional $\mathrm{K}$ Best SDA. In $[28,29]$, it is shown that the conventional $K$ Best SDA slightly outperforms the complex $K$-Best SDA and requires lower complexity. However, the conventional $K$-Best SDA may not always be applicable in some communications systems with special diversity features. Modified $K$-Best SDA aims to reduce decoding complexity but usually introduces performance degradation, which is more significant in the complex domain [30,31]. These prompt the development of a low-complexity and high-performance $K$-Best SDA directly operating in the complex domain.

\section{Proposed Sorting Algorithm and Hardware Architecture}

This section proposes a complex $K$-Best sphere decoder that achieves the same performance as the conventional $K$-Best SDA with lower complexity. As described in the previous procedural summary, the $K$-Best SDA involves three major operations: path evaluation, sorting, and candidate selection. In the following, new algorithms for sorting and candidate selection will be developed to achieve the reduction in computations. The path evaluation part remains unchanged so that the decoding performance of the $K$-Best SDA can be maintained.

3.1. Candidate Sequence Generator in Complex Plane. To search the symbols efficiently in the complex plane, it is useful to construct a table of candidate symbol sequences within a given region [14]. First, a primitive block is defined to be a square block bounded by $\{1+j, 1-j,-1+j,-1-j\}$. The complex plane can be regarded as consisting of a lot of primitive blocks placed at equal distances. In Figure 2, a received symbol is located at $y_{i}^{\prime \prime}$ surrounded by four nearest candidate symbols $41,42,49$, and 50 in the constellation diagram. A candidate symbol sequence, 49-50-41-42, can then be formed according to their distance from $y_{i}^{\prime \prime}$ in ascending order. Consider then the square area centered at the origin and surrounded by the candidate symbols 27 , 28,35 , and 36 . Shifting the symbols $41,42,49$, and 50 to the symbols $27,28,35$, and 36 , respectively, a location $y_{i, M}^{\prime \prime}$ corresponding to $y_{i}^{\prime \prime}$ can be identified. A new candidate symbol sequence, 35-36-27-28, can be identified likewise according to their distance from $y_{i, M}^{\prime \prime}$ in ascending order. Apparently the relation in terms of the distance from $y_{i}^{\prime \prime}$ to nearby candidate symbols remains unchanged after the coordination transformation. On the other hand, since the 


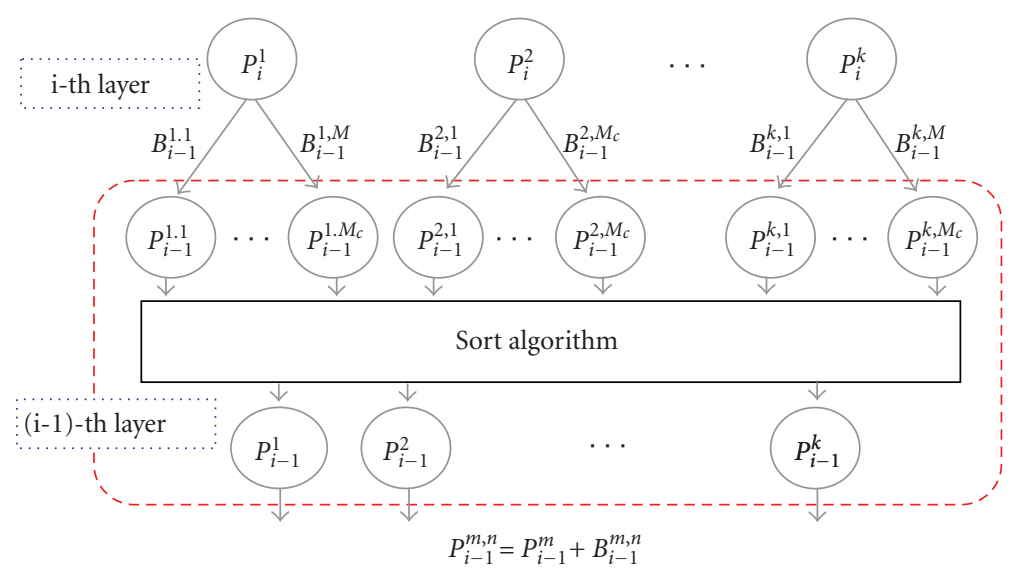

FIgURE 1: Illustration of the multi-index operation.

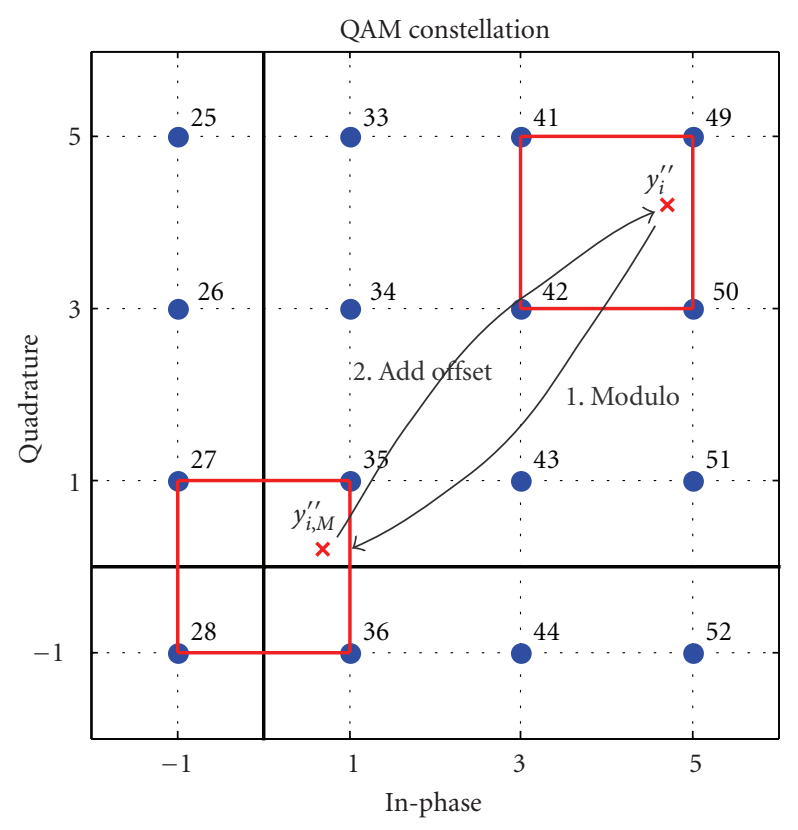

FIgURE 2: Modulo operation of the search center.

symbols are placed symmetrically in the complex plane, once the relation between a received symbol and the associated candidate symbol sequence in one of the four quadrants is obtained, those in the other three quadrants can be readily derived. Next, Figure 3 shows quadrant I of the solidline square area in Figure 2. The area is divided into 30 segments (we will explain how to partition the specified square area later). It can be verified that all symbols inside any given segment share the same candidate symbol sequence of $k$ symbols, where $k=11$ in Figure 3. For example, consider two symbols " $c$ " and " $d$ " inside segment 01 and evaluate the distances between all valid candidate symbols and the two points. It is straightforward to verify that the resulting two candidate sequences are identical, that is, $\{1+$ $j,-1+j, 1-j,-1-j, 1+j 3,-1+j 3,3+j,-3+j, 3-$ $j,-3-j, 1-j 3\}$. For other segments, the same result applies.

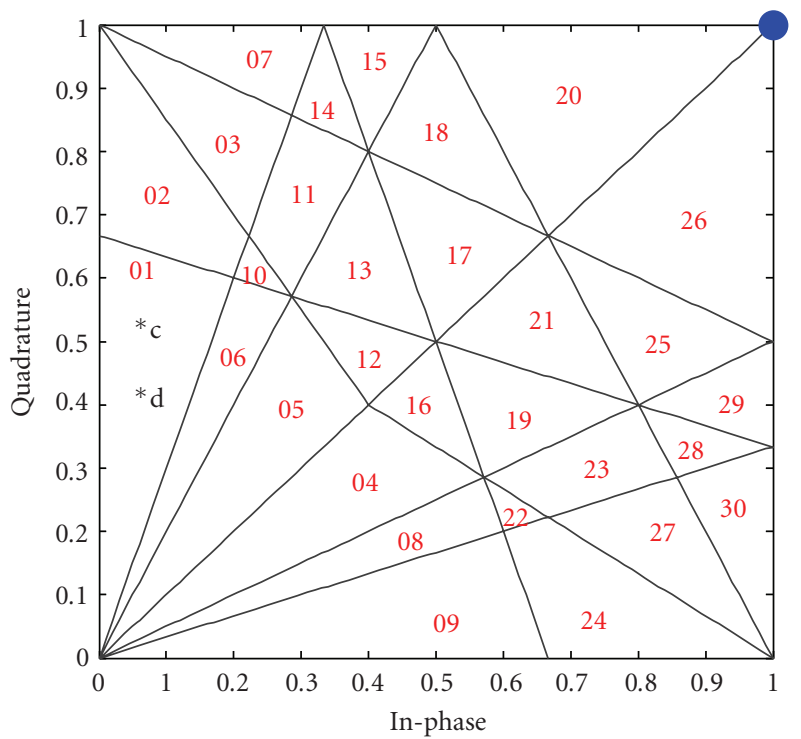

Figure 3: Partition of the search segments.

Using the above properties, we can construct a table of candidate symbol sequences of the $K$ nearest constellation symbols for all symbols bounded by $\{1+j, 1-j,-1+j,-1-$ $j$ \} instead of generating approximated path weights $[30,31]$. Due to the symmetry of 16-QAM and 64-QAM, a simple transformation allows symbols in the region bounded by $\{1+j, 1-j,-1+j,-1-j\}$ in quadrants II, III, and IV to use the same table as quadrant I. Any symbol located within the bounded region is first mapped to quadrant I by a simple transformation. The transformed result acts as the search center for finding the $k$ nearest candidate symbols by looking up the table of the symbol sequences, where $k$ is a specified number. When the candidate symbol sequence $\left\{x_{i}\right\}$ is found, it can easily be transformed back to the original quadrant. Figure 3 shows the partition of the search segments in quadrant $\mathrm{I}$, and the corresponding symbol sequences are listed in Table 1 , where $k=11$ is chosen as an example. This 
TABLE 1: List of candidate sequences.

\begin{tabular}{|c|c|}
\hline $\begin{array}{l}\text { Segment } \\
\text { ID }\end{array}$ & Candidate sequence \\
\hline 01 & $\begin{array}{c}1+j,-1+j, 1-j,-1-j, 1+j 3,-1+j 3,3+j,-3+ \\
j, 3-j,-3-j, 1-j 3\end{array}$ \\
\hline 02 & $\begin{array}{c}1+j,-1+j, 1-j,-1-j, 1+j 3,-1+j 3,3+j,-3+ \\
j, 3-j,-3-j, 3+j 3\end{array}$ \\
\hline$\vdots$ & $\vdots$ \\
\hline 29 & $\begin{array}{c}1+j, 1-j,-1+j, 3+j,-1-j, 3-j, 1+j 3,-1+ \\
j 3,3+j 3,1-j 3,-1-j 3\end{array}$ \\
\hline 30 & $\begin{array}{c}1+j, 1-j,-1+j, 3+j,-1-j, 3-j, 1+j 3,1- \\
j 3,-1+j 3,3+j 3,-1-j 3\end{array}$ \\
\hline
\end{tabular}

table can be constructed in advance by the following offline procedure:

First, the bounded square area by $\{1+j, 1, j, 0\}$ is divided into $u^{2}$ grids, by $(u-1)$ equally space horizontal and $(u-1)$ equally space vertical lines, where $u$ is chosen according to the required resolution. The corresponding distances between all valid candidate symbols and the center of each grid, which represents all possible received symbols within, are then evaluated. Next, by using some sorting procedure, the associated candidate sequence of any possible received symbol can be determined. Finally, all these possible symbols are rearranged into several search segments such that each segment has the same candidate sequence. By this approach, it is easy to tackle any predefined constellation rotation during run-time processing. The following describes the run-time operation in detail.

For any given search center $y_{i}^{\prime \prime}$ in the complex plane, the CSG first rounds it to the relative position $y_{i, M}^{\prime \prime}$ which lies inside the region bounded by $\{1+j, 1-j,-1-j,-1+j\}$. This modulo operation is depicted in Figure 2, and the associated relationship is described as follows:

for $\operatorname{Re}\left(y_{i}^{\prime \prime}\right)$

$$
\begin{gathered}
\text { X_offset }=\left\lfloor\operatorname{Re}\left(y_{i}^{\prime \prime}\right)\right\rfloor+\bmod \left(\left\lfloor\operatorname{Re}\left(y_{i}^{\prime \prime}\right)\right\rfloor, 2\right) \\
\operatorname{Re}\left(y_{i, M}^{\prime \prime}\right)=\operatorname{Re}\left(y_{i}^{\prime \prime}\right)-\text { X_offest }
\end{gathered}
$$

for $\operatorname{Im}\left(y_{i}^{\prime \prime}\right)$

$$
\begin{gathered}
\text { Y_offset }=\left\lfloor\operatorname{Im}\left(y_{i}^{\prime \prime}\right)\right\rfloor+\bmod \left(\left\lfloor\operatorname{Im}\left(y_{i}^{\prime \prime}\right)\right\rfloor, 2\right) \\
\operatorname{Im}\left(y_{i, M}^{\prime \prime}\right)=\operatorname{Im}\left(y_{i}^{\prime \prime}\right)-\mathrm{Y}_{\text {offest. }}
\end{gathered}
$$

Figure 4(a) shows the modulo unit of $\operatorname{Re}\left(y_{i}^{\prime \prime}\right)$ based on the 2 's complement property, which is efficiently implemented by a single adder and a few bit manipulations. $S$ is the sign bit (i.e., MSB) of $\operatorname{Re}\left(y_{i}^{\prime \prime}\right)$ and $b_{0}$ is the LSB of the integer part of $\operatorname{Re}\left(y_{i}^{\prime \prime}\right)$. Since the modulo operation of $\operatorname{Im}\left(y_{i}^{\prime \prime}\right)$ is the same as $\operatorname{Re}\left(y_{i}^{\prime \prime}\right)$, the modulo circuits of $\operatorname{Im}\left(y_{i}^{\prime \prime}\right)$ and $\operatorname{Re}\left(y_{i}^{\prime \prime}\right)$ are identical.

In the next step, if $y_{i, M}^{\prime \prime}$ lies in quadrant II, III, or IV, the CSG unit maps $y_{i, M}^{\prime \prime}$ into quadrant I by rotating $\pi / 2, \pi$ and $3 \pi / 2$, respectively. Figure 4 (b) shows this transformation circuit. The multiplexers chooses a right data path based on the sign bits of $\operatorname{Re}\left(y_{i, M}^{\prime \prime}\right)$ and $\operatorname{Im}\left(y_{i, M}^{\prime \prime}\right)$. The coordinates $d x_{-} t$ and $d y_{-} t$ denote the transformed values of $\operatorname{Re}\left(y_{i, M}^{\prime \prime}\right)$ and $\operatorname{Im}\left(y_{i, M}^{\prime \prime}\right)$, respectively.

The set $\left(d x_{-} t, d y_{-} t\right)$ is sent to the candidate generator unit to generate the desired candidate sequence $\left\{x_{i}\right\}_{i=1}^{k}$ using a table lookup operation. The contents of the segment identification (ID) and its corresponding candidate sequence are stored in ROM 1 and ROM 2, respectively, as shown in Figure 5, where the hardware architecture of the candidate generator is depicted. The found candidate symbol is first rotated into its original quadrant, and then the offset pair (X_offset, Y_offset) is added to the coordinates of the found candidate symbol. After the constellation restoration, the constellation boundary checker checks whether or not the found symbol lies inside the constellation boundary. If the found restored symbol is a legal one, the distance calculator calculates the value of $\left\|y_{i}^{\prime \prime}-x_{i}\right\|^{2}$. Multiplying the value of $\left\|y_{i}^{\prime \prime}-x_{i}\right\|^{2}$ by $r_{i, i}^{2}$ and adding the parent weight $P_{i+1}$ to the multiplied result, we obtain the path weight $P_{i}$ of the found symbol. The CSG can efficiently generate the coordination pairs of valid candidates and the associated path weights according to their path weights in an ascending order for each given received symbol. From Figure 5, the major hardware cost of the CSG involves 3 multipliers, 12 adders, and 2 ROMs. The ROM sizes (number of logic gates) are 2116 (ROM 1, with $u=32$ ) and 731 (ROM 2), respectively, according to the Synposys synthesis tools.

For any given symbol and its neighbors, which share the same candidate sequence, the candidate sequence is generated from the $k$ nearest constellation symbols by sorting their relative distance to the search center though these distance values are different for each different search center. The proposed CSG utilizes this property to generate a candidate sequence in ascending order and calculates the associated path weights so as to avoid a heavy load of path weight evaluations and sorting. Based on this concept, we can choose the appropriate $k$ to fit the system requirement. The ROM size expands quickly when a large value of $k$ is chosen. To remedy this, we can divide $k$ into a set $\left\{k_{i}\right\}_{i=1}^{p}$, where $\sum_{i=1}^{p} k_{i}=k$ such that the ROM can be kept at a realizable size.

3.2. Architecture of Highly Parallel Comparison Circuit (HPCC). The sorting operations in the $K$-Best decoder dominate the major complexity at each search layer. Hence, sorting is a critical factor in reducing the complexity of the $K$-Best SDA. The previously proposed CSG module can be applied to the $K$-Best SDA by exploiting the inherent partial orders coming with the property of CSG. This can be efficiently accommodated by applying the $K$-merge algorithm [30, 32]. For a more practical implementation, an efficient architecture that can effectively reduce the sorting complexity is needed.

Recall the definitions of branch weights and PED in Section 2. Let $P_{i}^{1}, P_{i}^{2}, \ldots, P_{i}^{K}$ denote the $K$ smallest PEDs in the $i$ th layer. After full-path expansion, we have $K M_{c}$ PEDs $P_{i-1}^{1,1}, P_{i-2}^{1,2}, \ldots, P_{i-1}^{1, M_{c}}, \ldots, P_{i-1}^{K, 1}, P_{i-2}^{K, 2}, \ldots, P_{i-1}^{K, M_{c}}$ at layer $i$, where $P_{i-1}^{m, n}$ stands for the PED of the $n$th path expanded from the 


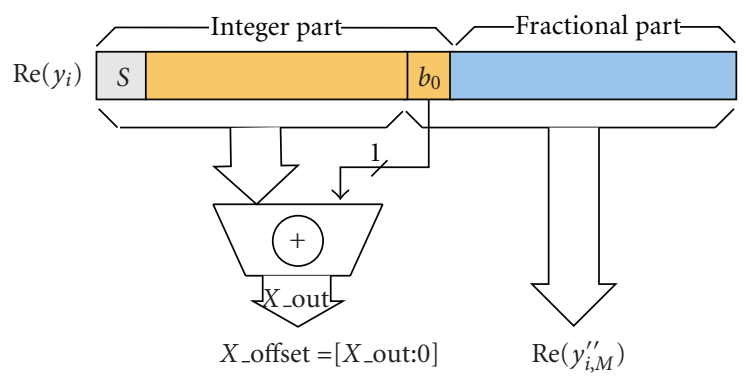

(a)

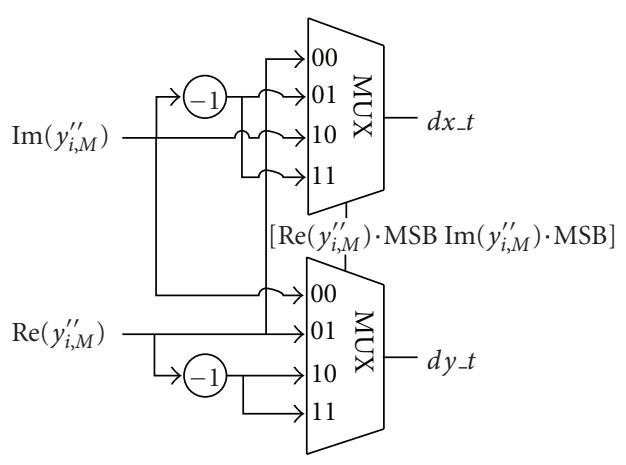

(b)

Figure 4: (a) Modulo unit of $\operatorname{Re}\left(\mathrm{y}_{\mathrm{i}}^{\prime \prime}\right)$. (b) Transformation unit of $\mathrm{y}_{\mathrm{i}, \mathrm{M}}^{\prime \prime}$.

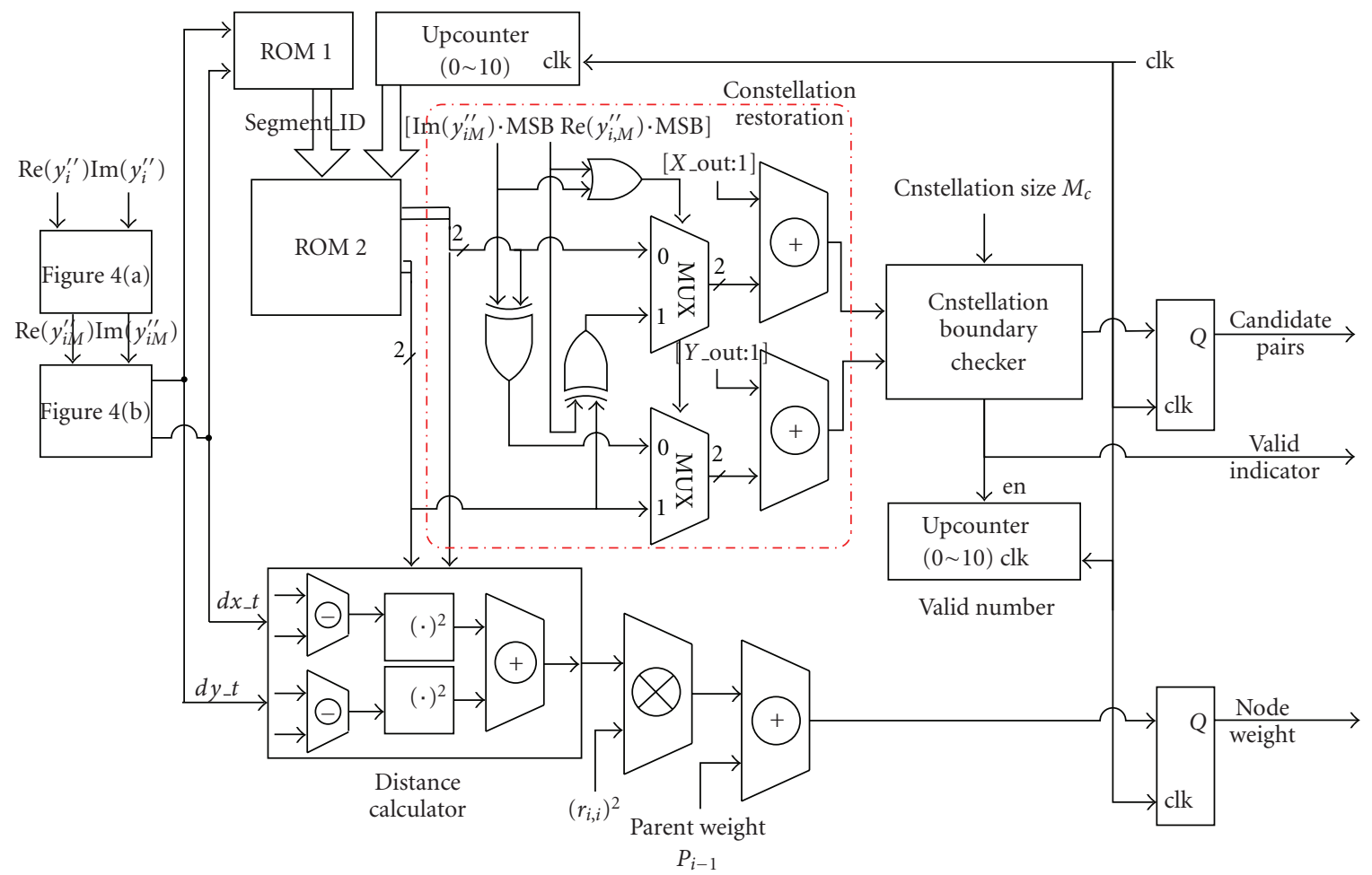

FIGURE 5: Hardware architecture of the candidate generator.

$m$ th parent node at layer $i$. Moreover, based on the sorted results from the $i$ th layer and the generated sequence from the proposed CSG module, we have $P_{i}^{1}<P_{i}^{2}<\cdots<$ $P_{i}^{K}$ and $P_{i-1}^{j, 1}<P_{i-1}^{j, 2}<\cdots<P_{i-1}^{j, k}$ for each $1 \leq j \leq$ $k$. Selecting the node with the smallest PED from the set $\left\{P_{i}^{1,1}, P_{i}^{2,1}, \ldots, P_{i}^{K, 1}\right\}$ is equivalent to finding the smallest PED from the full path expansion set containing $K M_{c}$ nodes. These operations are illustrated in Figure 6. Exploiting these properties instead of using traditional sorting algorithms, we can realize an efficient comparison architecture for the $K$ Best sorting at each stage that avoids full path evaluation and significantly reduces the sorting complexity. Figure 7 depicts this hardware architecture, and the following describes its operation.
The output sequence of the CSG module naturally forms a set in ascending order according to the evaluated PEDs while performing the $N$ th layer search. We, therefore, only need to conduct a single coordination transformation and $K$ path weight calculations. The generated results serve as the parent nodes of the next layer.

To search in the $(i-1)$ th layer, we first calculate $\left\{P_{i-1}^{1,1}, P_{i-1}^{2,1}, \ldots, P_{i-1}^{K, 1}\right\}$ and feed them into the HPCC. The candidate node with the smallest PED among these candidates is obtained immediately after $(K-1)$ compare-and-select (CAS) operations. If the chosen node comes from the $p$ th parent node, then the $P_{i}^{p}+B_{i-1}^{p, 2}$ PED is calculated, overwriting the previously chosen node. The node with the 2 nd smallest PED is obtained after $\log _{2} K$ CAS operations (only $\log _{2} K$ 


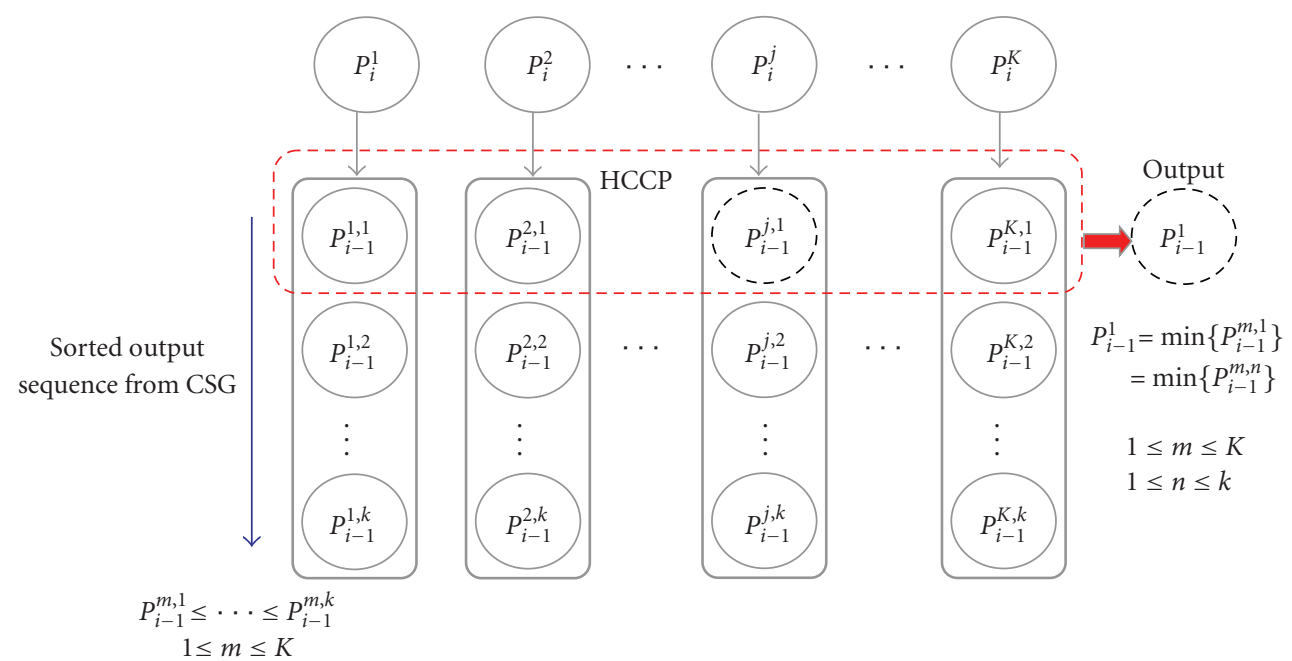

FIGURE 6: Illustration of the HPPC operations.
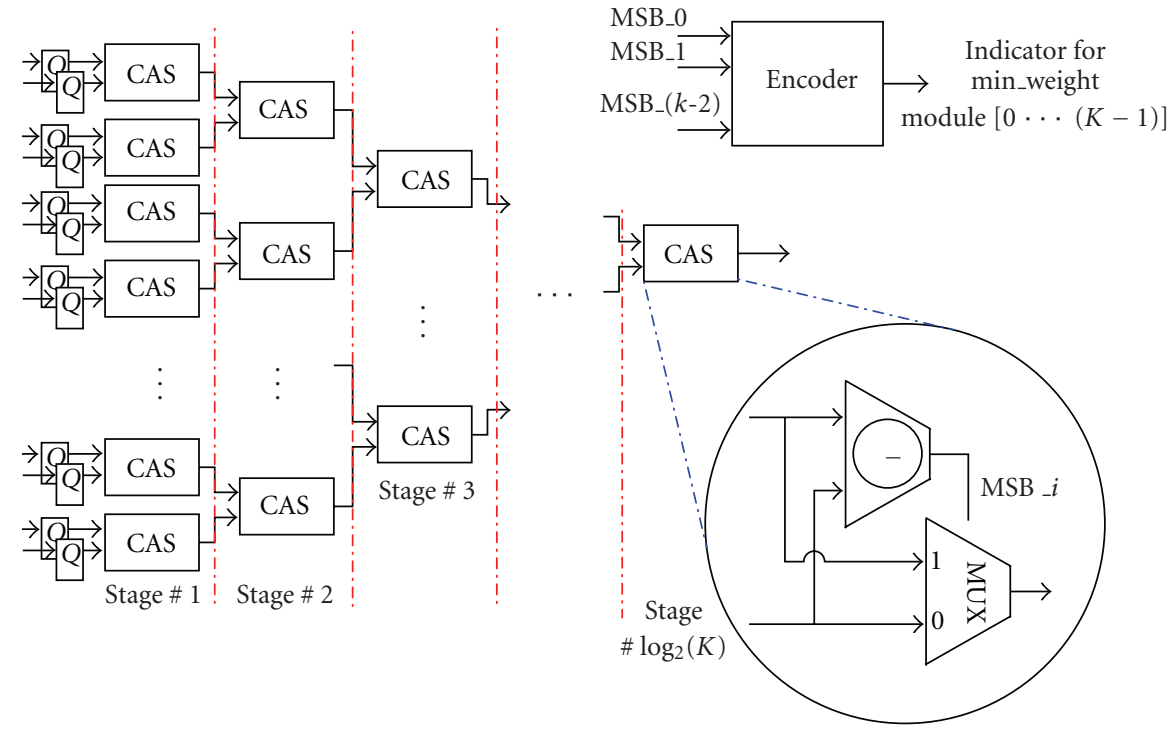

FIgURE 7: HPCC architecture.

results need be re-computed). Repeating this procedure, we can successfully select $K$ candidate nodes with the smallest PEDs from the entire valid candidate set. The survival set acts as the parent nodes of the $(i-2)$ th layer. In searching the nodes in each layer, we use $K$ coordination transformation, $(2 K-1)$ path weights evaluations, and $(K-1)\left(1+\log _{2} K\right)$ CAS operations. Note that the computational complexity of this approach is nearly fixed and independent of the constellation size $M_{c}$ of the transmitted symbols. Furthermore, the nodes in the survival set still exhibit an ascending order according to their PEDs. In the final search layer, that is, the 1st layer, we only need to choose the node with the smallest PED as the detection result. Hence, it takes only $K$ coordination transformation, $K$ PEDs evaluations, and $(K-1)$ CAS operations.
Compared with the winner path expansion method [33, 34], the proposed architecture, which is also frequently found in Viterbi decoder for choosing the minimal path metric, can avoid performing unnecessary operations thanks to the property of parallel computation. Moreover, it requires a smaller number of CAS $(K-1)$ than that of the conventional bubble sort method $(K)$.

3.3. Complexity Advantages. Through the combination of the two proposed modules, we only need $K$ coordination transformations, $(2 K-1)$ PED evaluations, and $(K-1)(1+$ $\log _{2} K$ ) CAS operations in each layer to obtain $K$ nodes with the smallest PEDs, regardless of the constellation size. These PEDs only need to be calculated when they are fed into the HPCC. Hence, the proposed architecture avoids exhaustive 
path weight evaluations as required in the conventional bubble sort architecture.

Previous methods attempt to reduce computational complexity by eliminating the number of visited nodes based on the probability or statistical properties of the additive noise. These methods provide an approximate solution and barter decoding performance for complexity reduction. As an alternative, this paper presents another way to reduce complexity with the premise of carrying on high-quality decoding results. The proposed approach utilizes operation decomposition, reconstruction, and associated efficient hardware architecture to select and evaluate only the most promising candidate symbols. The proposed method also significantly reduces computational complexity and provides an efficient solution with a nearly fixed throughput. These advantages are further enhanced when a larger constellation size is adopted. Although the proposed method incurs the extra cost of coordination transformation and restoration, it eliminates many path calculations and sorting operations and provides the same performance as the conventional $K$-Best SDA.

\section{Proposed Search Strategy for Near-ML Performance}

One way to reduce the complexity of the conventional $K$-Best SDA is to choose a smaller number of survival nodes in each layer. However, this can cause performance degradation in term of error rate. Instead of choosing a sufficiently large $K$ to achieve the near-ML performance, a new search strategy is proposed. The proposed search strategy preserves all candidate symbols and performs the ML search in the preceding layers when dealing with poor channel conditions. Only $K$ candidates are kept for the remaining lower layers. The following sections show how to determine the number of layers performing the ML search.

4.1. Preprocessing with Column Permutation. The channel matrix can be preprocessed with various techniques to reduce the complexity of candidate search and/or improve the performance of the $K$-Best SDA. Many preprocessing techniques can be used for this purpose, including column permutation [13], scaling [35], and lattice reduction [36]. In this paper, column permutation is adopted, in which the permutation order is determined according to the column norms of the channel matrix in ascending order. Given the QR decomposition of the ordered channel matrix $\mathbf{H}_{o}=$ $\mathbf{Q}_{o} \mathbf{R}_{o}$, we characterize below the cumulative distribution function (cdf) of the square of the diagonal entries of $\mathbf{R}_{o}$ denoted by $r_{o, i, i}^{2}$ (see the appendix):

for $i=1$

$$
\begin{aligned}
& F_{r_{o, i, i}^{2}}(r)=\int_{0}^{r} \frac{N !}{(N-1) !(M-1) !}\left[\sum_{k=0}^{M-1} \frac{x^{k}}{k !} e^{-x}\right]^{N-1} \\
& \cdot x^{M-1} e^{-x} d x
\end{aligned}
$$

for $2 \leq i \leq N$

$$
\begin{gathered}
F_{r_{o, i, i}^{2}}(r)=C_{i i} \int_{0}^{1} \int_{0}^{r / s}\left[1-\sum_{k=0}^{M-1} \frac{x^{k}}{k !} e^{-x}\right]^{i-1}\left[\sum_{k=0}^{M-1} \frac{x^{k}}{k !} e^{-x}\right]^{N-i} \\
\cdot x^{M-1} e^{-x}(s)^{M-i}(1-s)^{i-2} d x d s,
\end{gathered}
$$

where

$$
C_{i i}=\frac{N !}{(N-i) !(M-i) !(i-1) !(i-2) !} .
$$

Comparing (13)-(15) with the results of [13], the ordering mechanism increases $E\left[r_{i, i}^{2}\right]$ in the preceding layers, producing two main benefits. First, for a fixed $K$ in the $K$ Best SDA, increasing $E\left[r_{i, i}^{2}\right]$ in the preceding layers reduces the effective search range of the candidates. This in turn reduces the probability of the ML solution being dropped in the preceding layers. Another benefit is that it constrains the growth of the tree and hence reduces search complexity.

4.2. Proposed Search Strategy. For the Nth layer, the candidate symbol should satisfy the following search constraint according to (7):

$$
\left\|y_{N}^{\prime \prime}-x_{N}\right\|^{2} \leq\left(\frac{d^{\prime}}{r_{N, N}}\right)^{2} .
$$

Clearly, $1 / r_{N, N}$ will enlarge the constraint region when $r_{N, N}$ is smaller than 1 . In this case, the probability of the ML solution being dropped will increase when only $K$ nodes are kept in the $N$ th layer. To avoid performance degradation, conducting the ML search in the preceding layers [27] is one of the approaches usually adopted. To further reduce the computational complexity, we propose to perform the ML search in the $i$ th layer only when any $r_{i, i}$, where $N-L_{\mathrm{ML}}+1 \leq i \leq N$, is smaller than a given threshold $T_{r}$, with $L_{\mathrm{ML}}$ denoting the number of layers performing the ML search; the threshold $T_{r}$ will be decided later. This proposed search strategy is named conditional-ML (CML) search. Hence, the number of layers performing the ML search depends on the distribution of $r_{i, i}^{2}$. Figures $8(\mathrm{a})$ and $8(\mathrm{~b})$ show the impact of $r_{N, N}$ on the constrained search region. Based on the derived results in (13)-(15), we can systematically determine the number of layers performing the ML search under different $M$ and $N$.

Figure 9(a) shows the cdf curves of $r_{o, i, i}^{2}$ for the $4 \times 4$ MIMO channel. The probability of $r_{o, i, i}^{2}<1$ in the 4 th layer is larger than that in the other layers. Hence, only the 4 th layer needs to perform the ML search. Figure 9 (b) shows the cdf curves of $r_{o, i, i}^{2}$ for the $8 \times 8 \mathrm{MIMO}$ channel. In this case, the probabilities of $r_{o, i, i}^{2}<1$ in the 8 th and the 7 th layers are larger than that in the other layers. However, the number of possible candidates in the 7th layer is $\left(M_{c}\right)^{2}$ in the worst case, which is too large to store in a hardware implementation when $M_{c}$ is large. Hence, we keep all possible candidates in the 8th layer. For the 7th layer, we first find all possible candidates and keep $K$ survival nodes with the minimum path weights. 


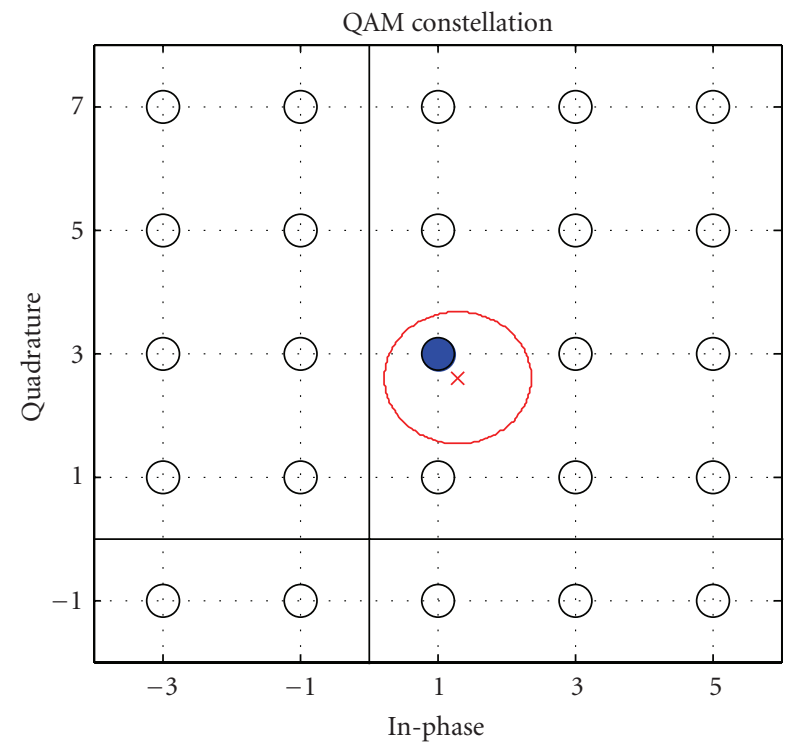

(a)

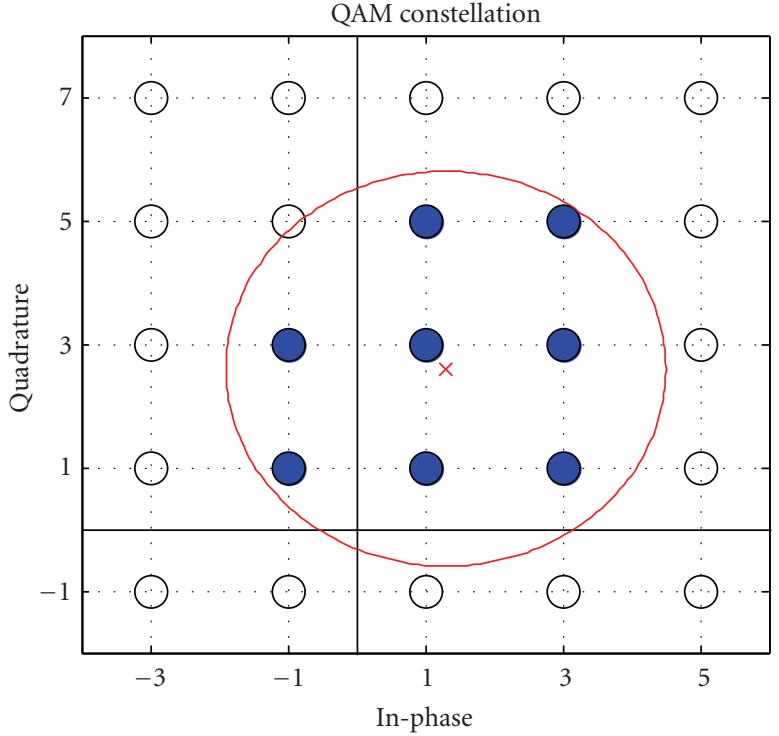

(b)

FIGURE 8: Search constraints of the Nth layer with $d^{\prime}=1$.1. (a) $r_{N, N}=1$. (b) $r_{N, N}=0.33$.

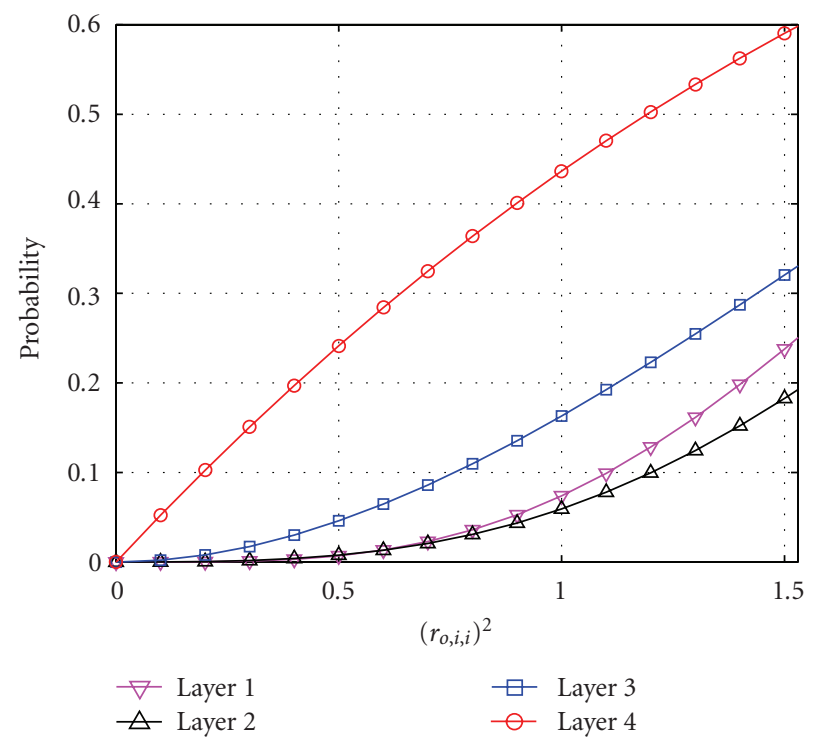

(a)

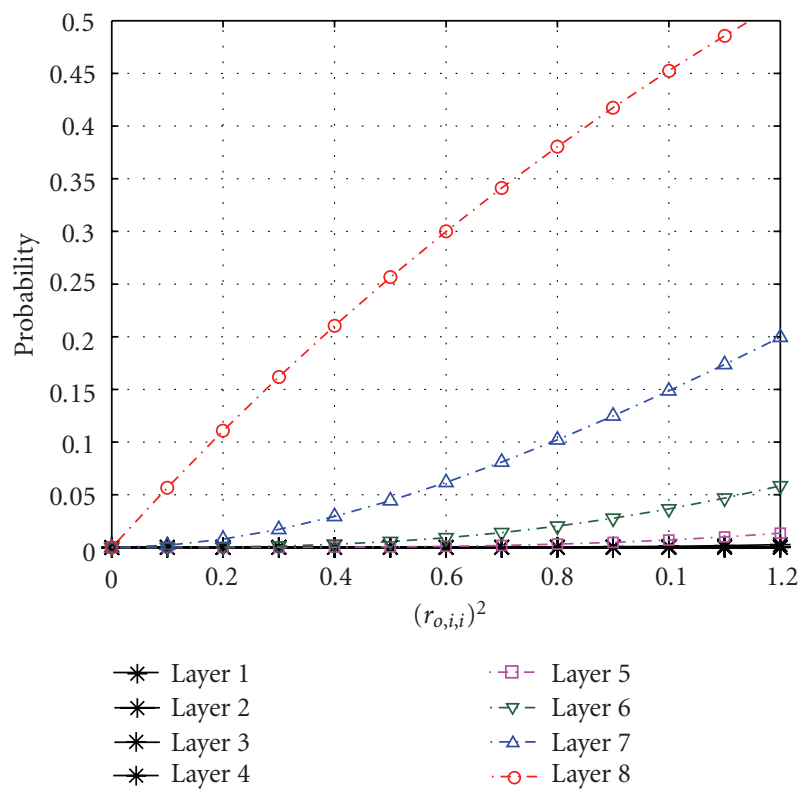

(b)

FIgURe 9: Cdf curves of $r_{o, i, i}^{2}$. (a) $4 \times 4$ MIMO channel. (b) $8 \times 8$ MIMO channel.

Next, we discuss how to decide the threshold $T_{r}$. Recall that for the $N$ th layer, a search for the candidate symbols should satisfy the following constraint:

$$
r_{N, N}^{2}\left\|y_{N}^{\prime \prime}-x_{N}\right\|^{2} \leq\left(d^{\prime}\right)^{2} .
$$

The proposed algorithm only keeps the $k$ constellation symbols nearest to $y_{N}^{\prime \prime}$ where $k=\min (K, 11)$; hence, the value of $\left\|y_{N}^{\prime \prime}-x_{N}\right\|^{2}$ has a limited range. Number 11 is chosen according to [37], which suggests that producing 11 candidate symbols yields quite good performance for practical applications. Therefore, we configure CSG to generate only 11 candidate symbols to reduce the implementation cost. From the previous argument and (17), it is straightforward to see that the threshold $T_{r}$ can be chosen based on the following criterion:

$$
\begin{gathered}
T_{r} D \geq \mathrm{E}\left[\left(d^{\prime}\right)^{2}\right], \\
D=\min \left(D_{K}, D_{11}\right),
\end{gathered}
$$


where $D_{K}$ and $D_{11}$ denote the distances from the $K$ th and 11th nearest constellation symbols to $s_{N}$, respectively. $E\left[\left(d^{\prime}\right)^{2}\right]$ is the expected value taken with respect to the channel statistics; it is used in place of $\left(d^{\prime}\right)^{2}$ because $d^{\prime}$ is typically a random variable depending on the channel condition and SNR [10, 13].

By using (18), when the $k$ nearest constellation symbols do not cover all the symbols inside the circle with a radius of $E\left[\left(d^{\prime}\right)^{2}\right]^{1 / 2}$, the ML search can be activated to retain all valid symbols. However, this will incur complexity increase because the probability of performing the ML search increases. The threshold $T_{r}$ thus acts as a tradeoff parameter between complexity and performance. Since $E\left[\left(d^{\prime}\right)^{2}\right]$ varies with SNR, we can choose $E\left[\left(d^{\prime}\right)^{2}\right]$ corresponding to the SNR at which the symbol error rate of the proposed $K$ Best SDA deviates from that of the ML detector by a certain normalized amount $\delta$. This ensures that the performance of the proposed $K$-Best SDA can be made close to ML detection. When applying the criterion in (18) to the $(N-1)$ th layer and below, the obtained threshold is sure to be smaller than the threshold of the $N$ th layer because the distance contributed by the Nth layer is a positive value. Thus, we can use the threshold of the $N$ th layer for other layers. In summary, the proposed CML search strategy only needs to check whether the values of $r_{o, i, i}$, that are already available from the QR decomposition, are smaller than $T_{r}$. It is not necessary to design any extra circuits to estimate the channel conditions for adjusting $K$ as in [24].

By the proposed criterion in (18)-(19), the system performance is insensitive to the choice of $K$ and the number of candidates generated by the CSG module. This is in contrast to the conventional $K$-Best SDA, in which the value of $K$ must be large enough, usually close to the constellation size $M_{c}$, to archive near-ML performance. Using the proposed criterion with the self-adjustment mechanism, the proposed $K$-Best SDA can choose a smaller $K$, as small as a half of $M_{c}$, to archive near-ML performance. In fact, when a smaller value of $K$ is chosen or the GSC module generates a shorter candidate sequence, the ML search will be activated more frequently trying to retain the possible ML solution.

The overall complexity of the proposed $K$-Best SDA can be predicted based on the complexity of the adopted sorting architecture, CML search procedure, choice of the value of $K$, the number of generated candidates of the CSG module, and the activation probability of the ML search. The decoder can thus achieve near-ML performance under a given complexity constraint without requiring a large value of $K$.

4.3. Joint 2-Layer ML Search Algorithm. According to the derived cdf of $r_{o, i, i}^{2}$ in (13)-(15) and observation in the previous sections, we only need to conduct a 2-layer full search in the worst case, which involve choosing $K$ survival nodes with the smallest path weights among $\left(M_{c}\right)^{2}$ nodes. In the original 2-layer ML search in the complex plane, for any received symbol, we need to evaluate all accumulated path weights between the search center and all valid candidate symbols while performing a full search of two layers. We then select $K$ nodes with the smallest accumulated path weights.

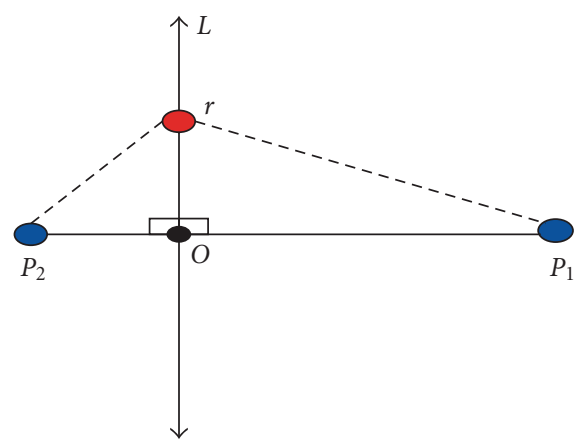

FIGURE 10: Geometrical relationship illustrating the adopted property for computation reduction.

Using the previously developed CSG and HPCC modules, we here propose an efficient procedure which only requires a minor modification of the control path of the HPCC. This modified procedure significantly reduces the computational burden and hardware implementation cost. The following describes the proposed procedure in detail.

First, for any received symbol in the complex plane, we evaluate the distances between the received symbol and all valid candidate symbols which lie on the same row of the square lattice before starting the sorting procedure. Consider the geometry shown in Figure 10. Let $r$ lie on line $L$, which is perpendicular to $\overline{p_{1} p_{2}}$ and intersects with $\overline{p_{1} p_{2}}$ at $o$. It is easy to show that if $\overline{o p_{1}}>\overline{o p_{2}}$ then $\overline{r p_{1}}>\overline{r p_{2}}$. Using this property, these candidate symbols can be ordered by their coordinate values on the $\mathrm{x}$-axis following the SE enumeration rule [12]. Therefore, a table containing the row vectors of sorted candidate symbols for each $x$ value would suffice and efficiently simplifies the sorting process [38].

Second, for any square lattice with $M_{c}$ symbols, these candidate symbols can be divided into $\sqrt{M_{c}}$ groups. Each symbol in the same group has the same $y$-axis coordinate value. Based on the prepared table, the procedure can efficiently generate $\sqrt{M_{c}}$ groups. Each group contains $\sqrt{M_{c}}$ sorted candidate symbols for each received signal without any extra sorting operation. The structure of each sorted group is the same as the output sequence of the proposed CSG module in ascending order. Therefore, utilizing the HPCC, after $\sqrt{M_{c}}+K-1$ path evaluations and $\left(\sqrt{M_{c}}-1\right)+$ $(K-1) \log _{2} \sqrt{M_{c}}$ CAS operations, the proposed procedure can generate $K$ candidates with the smallest PEDs for each given received symbol. These selected $K$ symbols are again arranged in ascending order according to their PEDs. Note that only these promising candidates are considered and completely evaluated. Finally, when a full search of two layers is required, it is only necessary to repeat $M_{c}$ times for $M_{c}$ possible parent symbols to generate a total of $K M_{c}$ promising symbols and divide them into $M_{c}$ sorted groups. Each group is sorted in ascending order according to the evaluated PEDs. The HPCC is then utilized to choose the $K$ survival symbols with the smallest PEDs. This can be done efficiently thanks to the naturally ascending order of each group.

The above procedure can achieve the same result as the ML exhaustive search, but its complexity is significantly 
TABLE 2: Computational complexity of proposed $K$-Best SDA (excluding interference cancellation).

\begin{tabular}{|c|c|c|c|c|c|}
\hline & \multicolumn{2}{|c|}{ ML search deactivated } & \multicolumn{3}{|l|}{ ML search activated } \\
\hline & $\begin{array}{c}\text { 1st layer } \\
\text { search }\end{array}$ & 2nd layer search & $\begin{array}{l}\text { Joint 2-layer ML } \\
\text { search }\end{array}$ & $\begin{array}{c}3 \mathrm{rd} \sim(\mathrm{N}-1) \text { th } \\
\text { layer search }\end{array}$ & $\begin{array}{c}\text { Nth layer } \\
\text { search }\end{array}$ \\
\hline CAS & 0 & $(K-1)\left(1+\log _{2} K\right)$ & $\begin{array}{l}M_{c}(K-1) \log _{2}\left(\sqrt{M_{c}}\right)+ \\
M_{c}\left(\sqrt{M_{c}}-1\right)+ \\
m(2 K-1)+(m- \\
1)\left[\log _{2}(m)-1\right]\end{array}$ & $(K-1)\left(1+\log _{2} K\right)$ & $(K-1)$ \\
\hline $\begin{array}{l}\text { Path } \\
\text { Accumulation }\end{array}$ & 0 & $(2 K-1)$ & $M_{c}\left(\sqrt{M_{c}}+K-1\right)$ & $(2 K-1)$ & K \\
\hline Path calculation & K & $(2 K-1)$ & $M_{c}+M_{c}\left(\sqrt{M_{c}}+K-1\right)$ & $(2 K-1)$ & K \\
\hline
\end{tabular}

Where $m=\left[M_{c} / k\right]$, if $\bmod \left(M_{c}, k\right)=0$ and $m=\left[M_{c} / k\right]+1$, otherwise.

TABLE 3: Computational complexity of conventional $K$-Best SDA in real domain (excluding interference cancellation).

\begin{tabular}{lccc}
\hline & $\begin{array}{c}\text { 1st layer } \\
\text { search }\end{array}$ & $\begin{array}{c}\text { 2nd } ~ \\
(2 N-1) \text { th } \\
\text { layer search }\end{array}$ & $\begin{array}{c}2 N \text { th layer } \\
\text { search }\end{array}$ \\
\hline CAS & $K \sqrt{M_{c}}$ & $K^{2} \sqrt{M_{c}}$ & $\left(K \sqrt{M_{c}}-1\right)$ \\
Path Accumulation & 0 & $K \sqrt{M_{c}}$ & $K \sqrt{M_{c}}$ \\
Path calculation & $\sqrt{M_{c}}$ & $K \sqrt{M_{c}}$ & $K \sqrt{M_{c}}$ \\
\hline
\end{tabular}

reduced when $K$ is small. The procedure fully reutilizes the previously proposed hardware architecture as described in Section 3 except an extra memory is required for the intermediate storage. It also inherits the advantages of CSG and HPCC which avoid the heavy load in path weight computation and sorting.

Tables 2 and 3 show the detailed computational complexity of the proposed $K$-Best SDA and the conventional $K$-Best SDA, respectively. Comparing the two tables, the required complexity of proposed $K$-Best SDA is approximately $\sqrt{M_{c}}$ times lower than the conventional one and is insensitive to the constellation size $M_{c}$, as mentioned earlier in Section 3.2. Although extra computations are needed when the CML search is activated in the proposed SDA, the probability of activation is small, as long as the channel is not severely ill conditioned. Therefore, the total required complexity of the proposed $K$-Best SDA can still be kept lower than that of the conventional SDA. As a final remark, since the proposed $K$ Best SDA can work with a smaller number of search layers and smaller value of $K$, compared to the conventional $K$-Best SDA, it has the potential of reducing the decoding latency of the latter because the decoding latency mainly depends on the number of search layers and required processing time per search layer, which in turn depends on $K$.

\section{Computer Simulations and Discussions}

This section simulates the symbol error rate (SER) performance and complexity of the proposed $K$-Best SDA and compares it with the SE SDA and conventional $K$-Best SDA [17]. Although many variants of the $K$-Best SDA have been proposed, the conventional one has the best decoding performance and is chosen here as a benchmark. For a fair comparison in each simulation, the preprocessing technique mentioned in Section 4.1 is applied to all algorithms. Complexity is measured in terms of the average number of floating point operations (flops). All real additions, multiplications, memory read/write, and comparisons are equally treated as flops. We set $d^{\prime}$ as the distance between the Babai estimate and the received signal [10], and $\mathrm{E}\left[\left(d^{\prime}\right)^{2}\right]$ is then obtained in advance for each SNR as the average from 100000 independent trials. In each simulation, we generate 100 noise realizations per channel realization, and at least 5000 channel realization for each SNR value. The SER is obtained as the average from 500000 independent trials.

We first investigate the effectiveness of the proposed CML strategy by comparing the performance of the complex $K$ Best SDA, which is mentioned in Section 2, with various configurations. An extreme value of $K=4$ is chosen for the complex $K$-Best SDA incorporating CML. Note that $K=4$ is in general the maximal acceptable value for a MIMOOFDM system, where the ML solution needs to be obtained for each subcarrier. The normalized deviation of SER is set as $\delta=15 \%$, the threshold $T_{r}$ is set as 0.42 according to (18), and the corresponding probability of performing the ML search is $9.08 \%$, according to (13)-(15). On the other hand, the $K$ values of the conventional complex $K$-Best SDA without CML are chosen as $K=4,8,12$ for $4 \times 416$-QAM, and $K=4,12,24$ for 64-QAM, respectively, to illustrate the performance difference. Figures 11(a), 11(b) show the $4 \times 4$ 16-QAM and 64-QAM simulations of SER respectively. From the results, the complex $K$-Best SDA incorporating CML can significantly improve the decoding performance with a small $K$ value. The reason is that the proposed CML strategy keeps all possible candidates in the first search layer when the channel is in a poor condition, significantly reducing the probability of the ML solution being dropped. The conventional complex $K$-Best SDA needs to choose $K=$ 12 and 24, respectively, to achieve the similar performance. Such high $K$ configurations would inevitably increase the computational complexity, decoding latency and infeasibility for practical MIMO-OFDM systems. For demonstration, we also include the cases of $K=8$ and $K=12$ for the complex 
$K$-Best SDA incorporating CML for 16-QAM and 64-QAM respectively. It is evident that both can achieve nearly the same performance as the ML detector.

Next, we evaluate the SER performance and complexity of the proposed complex $K$-Best SDA incorporating the CML strategy. Figures 12(a), 12(b) and 13(a), 13(b), respectively, show the $4 \times 4$ 16-QAM and 64-QAM simulations of SER and complexity with $K=8$. The normalized deviation of SER is set as $\delta=5 \%$ and $\delta=10 \%$, respectively, and the threshold $T_{r}$ is set as 0.291 and 0.3532 , respectively, according to (18), and the corresponding probability of performing the proposed ML search is $4.26 \%$ and $5.62 \%$, respectively, according to (13)-(15). Comparing Figure 12(a) with Figure 13(a), the SER curves of the proposed $K$-Best SDA and the SE SDA are nearly the same. This shows that the threshold constraint significantly reduces the probability of performing the ML search, and there is almost no performance degradation in the proposed $K$-Best SDA. In contrast, the SER of the conventional $K$-Best SDA tends to become saturated at high SNR. This is due to the fact that the conventional $K$-Best SDA with a smaller $K$ drops the ML solution with a high probability when the channel is in poor conditions, which always occurs with a certain probability in practice. In the 64-QAM case, the proposed $K$-Best SDA achieves nearly a $3 \mathrm{~dB}$ gain over the conventional $K$-Best SDA at SER $=10^{-3}$. Note that this performance gap between the proposed $K$-Best SDA and a conventional one is larger than that of the 16-QAM case. This is because the probability of the ML solution being dropped increases as the modulation symbol alphabet becomes larger [17]. In contrast, the proposed CML search strategy keeps all possible candidates in the preceding layers, significantly reducing the probability of the ML solution being dropped. Comparing Figure 12(b) with Figure 13(b), the proposed $K$-Best SDA has higher complexity than that of the SE SDA in the high SNR regime. This is due to the fact that the proposed $K$ Best SDA visits more candidate symbols than the SE SDA when the number of layers $N$ is smaller. As shown in the simulation cases, the complexity of the SE SDA varies with SNR. This is not desirable in practice, because a steady SNR is not achievable in realistic wireless environments, such that the decoding throughput of the SE SDA cannot be stable. In contrast, the proposed $K$-Best SDA provides a nearly fixed throughput, with excellent performance and low complexity. The proposed efficient architecture reduces the number of path weight evaluations and sorting operations in each layer. As a result, the proposed $K$-Best SDA exhibits near-ML performance and reduces $46.62 \%$ and $58.14 \%$ complexity, respectively, over the conventional $K$-Best approach using the same $K$.

Figures 14(a) and 14(b) show the $8 \times 8$ 16-QAM simulations of SER and complexity with $K=14$. The normalized deviation of SER is set as $\delta=15 \%$ and the threshold $T_{r}$ is set as 0.833 , and the corresponding probability of performing the proposed ML search is $43.9 \%$. The probability of performing the ML search is higher than that of the $4 \times 4$ case because the probability of the ML solution being dropped in the $K$-Best SDA is higher in the $8 \times$ 8 case. Again, the performance of the proposed $K$-Best SDA is better than the conventional $K$-Best SDA, and the complexity of the proposed $K$-Best SDA is lower than that of the SE SDA and the conventional $K$-Best SDA. Compared with the conventional $K$-Best SDA, the proposed method decreases complexity by more than $41.75 \%$. Because the number of search layers is larger in this case, the proposed method reduces more complexity in path evaluations and sorting operations. In the $8 \times 8$ case, the value of $K$ must be set larger to reduce the probability of the ML solution being dropped in the preceding layers. Hence, the gap in complexity between the proposed $K$-Best SDA and the conventional $K$-Best SDA is smaller than that in the $4 \times 4$ case. We can further improve the performance of the proposed $K$-Best SDA by choosing a higher threshold.

Figures $15(\mathrm{a})$ and $15(\mathrm{~b})$ show the $8 \times 864$-QAM simulations of SER and complexity with $K=32$. The normalized deviation of SER is set as $\delta=15 \%$ and the threshold $T_{r}$ is set as 1.143 and the corresponding probability of performing the ML search is $65.8 \%$. In this case, the proposed $K$-Best SDA still works better than the conventional $K$-Best SDA. The gap in complexity between the proposed $K$-Best SDA and the conventional $K$-Best SDA is smaller than that in the $8 \times 816$-QAM case. This is because a higher threshold value causes the ML search operations to occur more frequently though the proposed efficient sorting method reduces much more complexity for a larger modulation alphabet. The proposed CML search procedure significantly reduces the amount of path evaluations but induces extra memory read/write and table access operations. Nevertheless, the proposed $K$-Best SDA still has lower average complexity than the conventional one. Finally, under the same channel conditions, the conventional $K$-Best SDA requires $K=52$ to achieve near-ML performance. The configuration increases the computational complexity and decoding latency. The proposed decoder with $K=32$ can provide nearly the same performance, reducing $53.45 \%$ computational complexity over the conventional $K$-Best SDA with $K=52$.

This section simulates the SER performance and complexity of the proposed SDA and compares it with the SE SDA and the conventional $K$-Best SDA. Although the value of $r_{o, i, i}$ does not directly reflect the channel condition in all cases, the proposed criterion does help the decoder successfully produce a near ML solution over poor channels, without always performing the ML search in the preceding layers. This systematic approach thus requires fewer ML search layers than previous methods $[23,27]$. The simulation results confirm that the proposed decoder exhibits excellent performance and requires lower complexity than the conventional $K$-Best SDA. It is also worth noting that the performance of the proposed decoder is close to that of the SE SDA (i.e., ML performance).

\section{Conclusions}

In this paper, we propose a modified $K$-Best SDA with a new sorting algorithm and search strategy to achieve near-ML performance with low complexity. In conventional $K$-Best SDA, path-weight evaluation and sorting operations for all valid candidate symbols comprise the major computational 


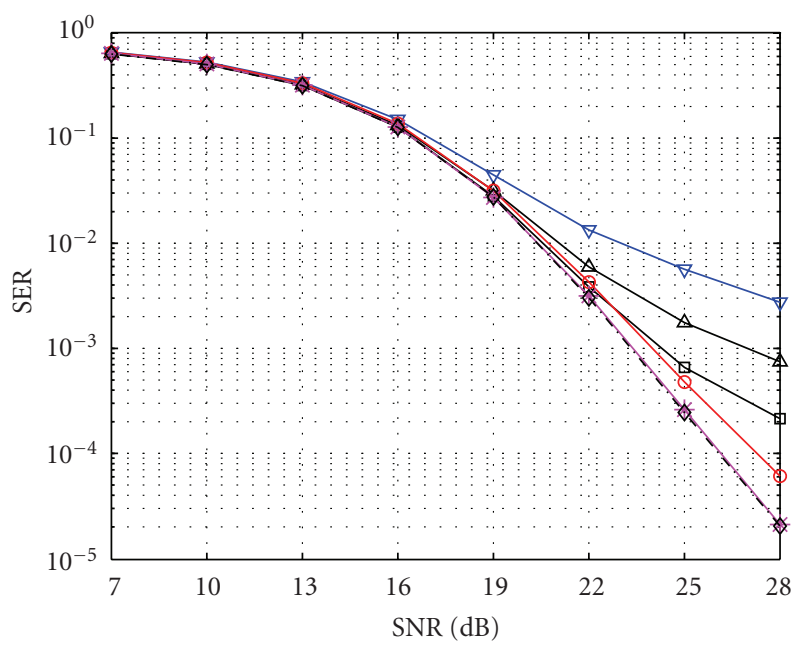

$\rightarrow$ Complex $K$-Best SDA without proposed CML $(K=4)$
$\triangle$ Complex $K$-Best SDA without proposed CML $(K=8)$
$\square-$ Complex $K$-Best SDA without proposed CML $(K=12)$
- Complex $K$-Best SDA with proposed CML $(K=4)$
$*$ Complex $K$-Best SDA with proposed CML $(K=8)$
$-\diamond-\operatorname{MLD}(4 \times 4)$

(a)

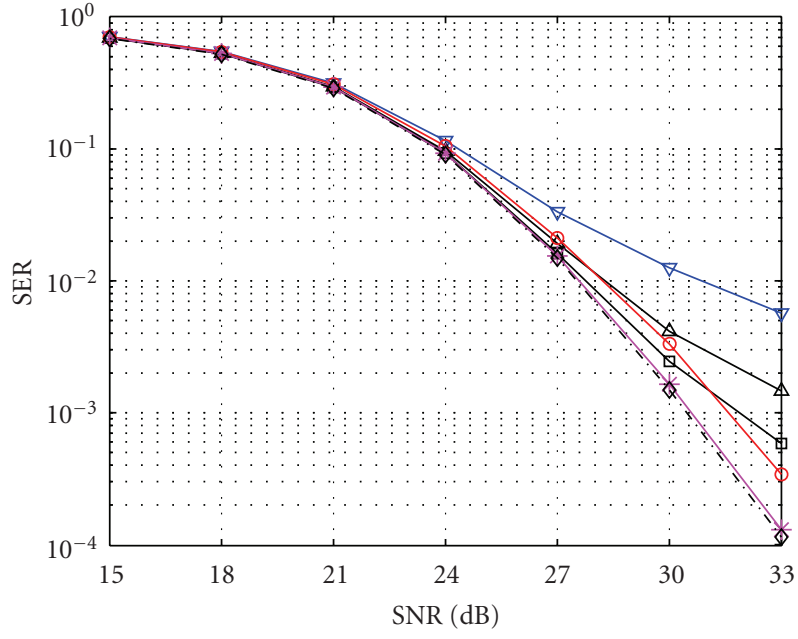

$\rightarrow$ Complex $K$-Best SDA without proposed CML $(K=4)$
$\triangle$ C Complex $K$-Best SDA without proposed CML $(K=12)$
$\square-$ Complex $K$-Best SDA without proposed CML $(K=24)$
- Complex $K$-Best SDA with proposed CML $(K=4)$
$*$ Complex $K$-Best SDA with proposed CML $(K=12)$
$-\diamond-\operatorname{MLD}(4 \times 4)$

(b)

Figure 11: Performance of complex $K$-Best SDA for $4 \times 4$ MIMO systems. (a) 16-QAM modulation. $K=4$ and 8 for complex $K$-Best SDA incoporating proposed CML strategy; $K=4 K, 8$, and 12 for regular complex $K$-Best SDAs. (b) 64 -QAM modulation. $K=4$ and 12 for complex $K$-Best SDA incoporating proposed CML strategy; $K=4 K, 12$, and 24 for regular complex $K$-Best SDAs.

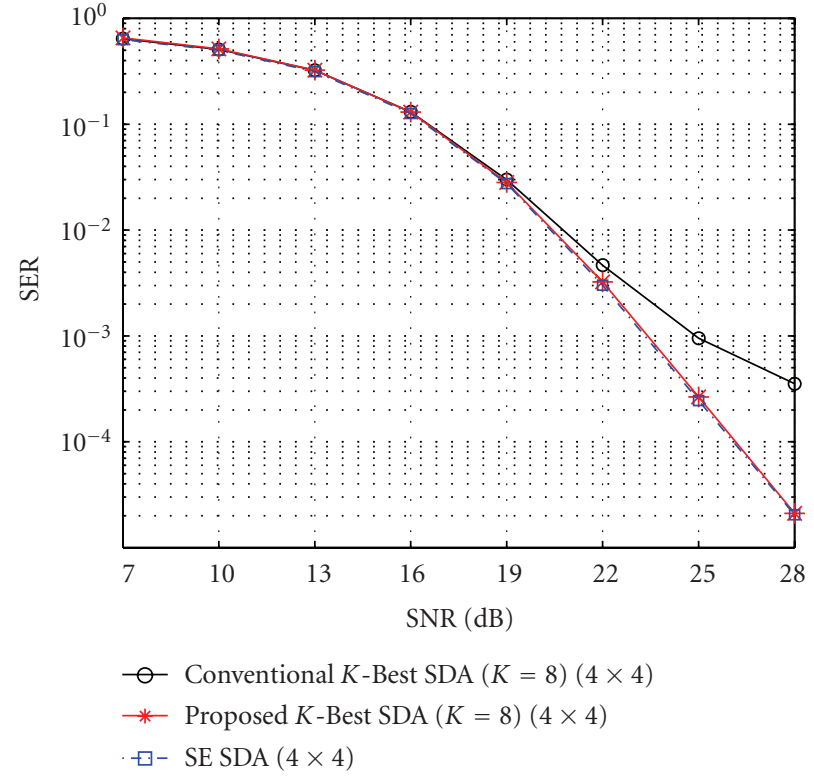

(a)

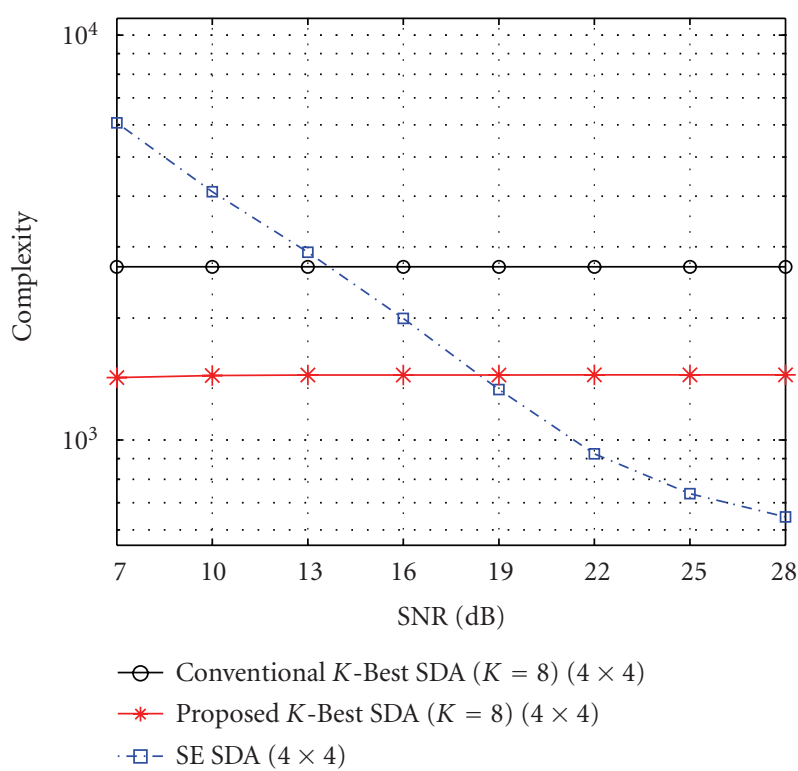

(b)

FIGURE 12: Performance and complexity of SDA for $4 \times 4$ MIMO systems with 16 -QAM modulation. (a) SER. (b) Complexity. $K=8$ for K-Best SDAs.

cost in each search layer. The new CSG generates candidate sequences in the complex plane instead of producing actual path weights, thus making it possible for the child nodes of each parent node to be sorted without any extra effort. Combining the CSG with a highly parallel comparison circuit, the proposed SDA can reduce computational complexity, while maintaining the same performance as the conventional $K$-Best SDA. To further improve decoding performance and efficiency, the new search strategy performs the ML search at a few preceding layers. A judicious criterion is 


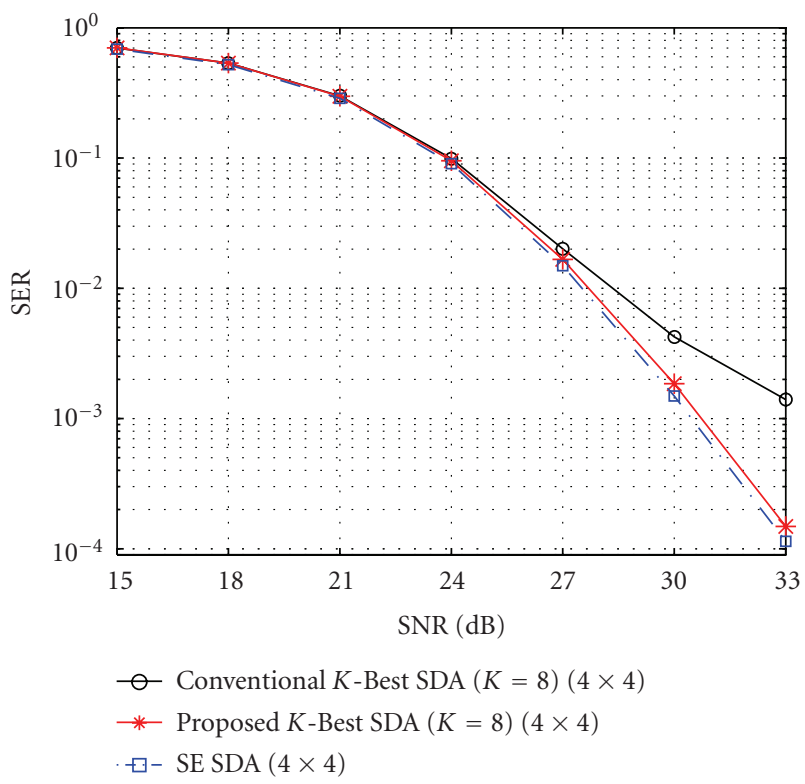

(a)

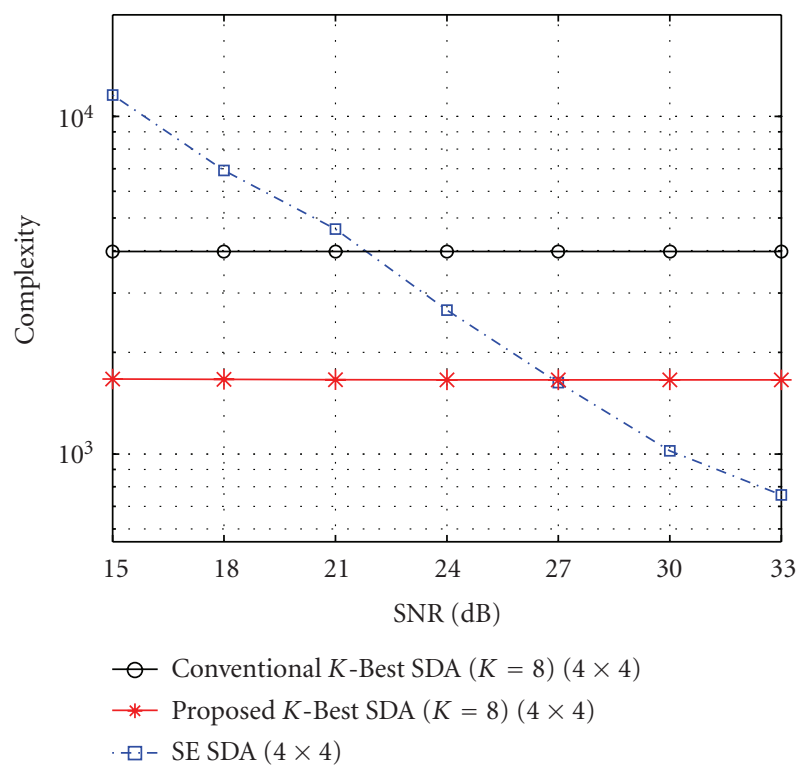

(b)

FIGURE 13: Performance and complexity of SDA for $4 \times 4$ MIMO systems with 64-QAM modulation. (a) SER. (b) Complexity. $K=8$ for $K$-Best SDAs.

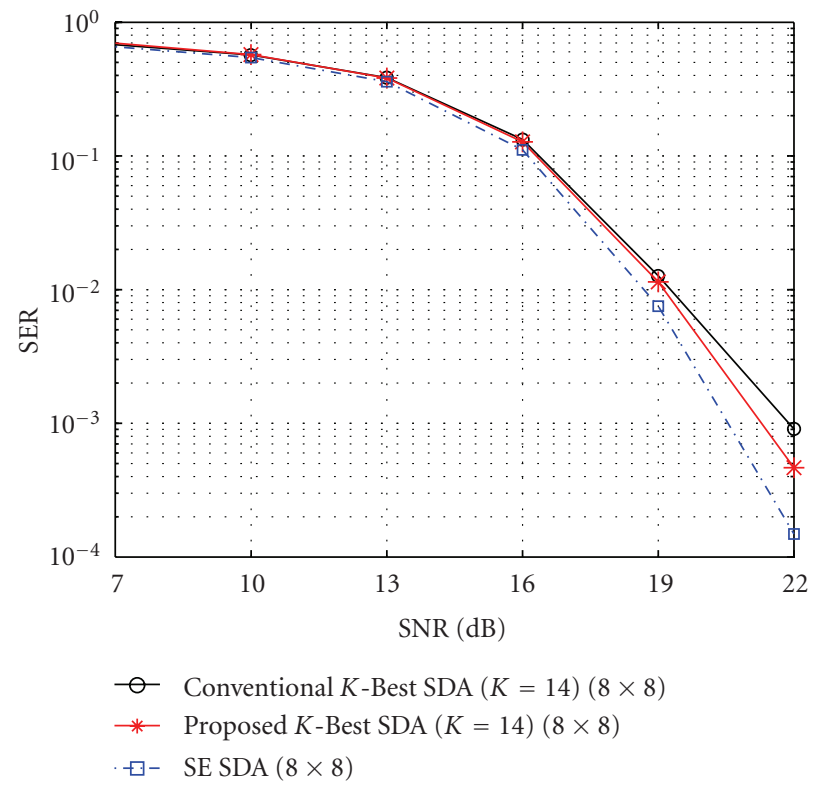

(a)

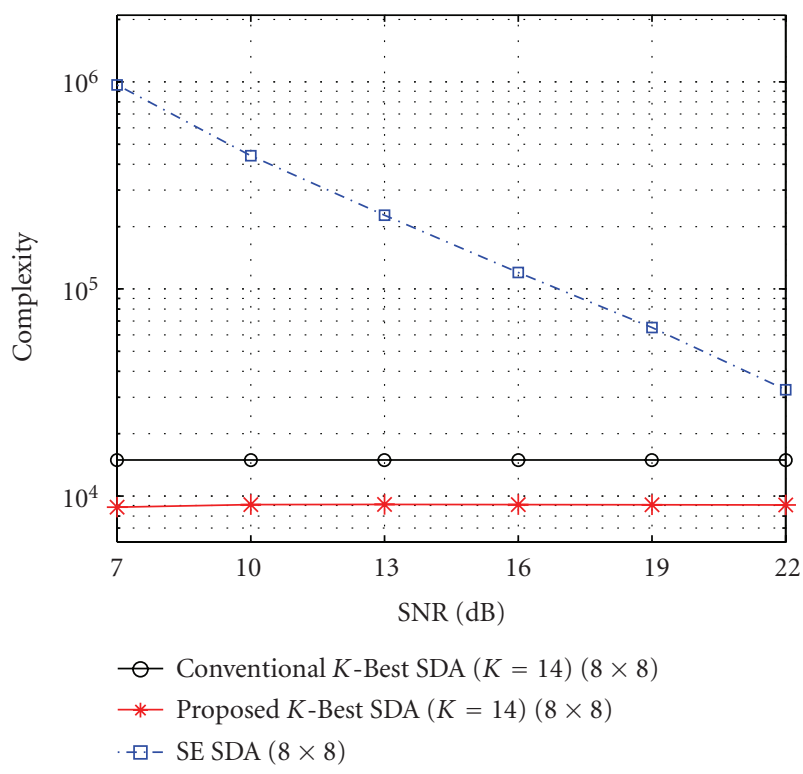

(b)

FIgure 14: Performance and complexity of SDA for $8 \times 8$ MIMO systems with 16-QAM modulation. (a) SER. (b) Complexity. $K=14$ for $K$-Best SDAs.

proposed accordingly to determine when to activate the ML search. Simulation results show that the proposed SDA effectively reduces the complexity of the conventional $K$-Best SDA, while offering superior SER performance at the high SNR regime. Its decoding performance is close to the ML performance even when the chosen value of $K$ is small.
To facilitate practical applications of the proposed SDA, a corresponding hardware architecture is also proposed. The architecture is quite regular and utilizes standard hardware elements without any extra complicated computational modules. As such, the proposed SDA is suitable for realtime applications and provides a promising solution for next 


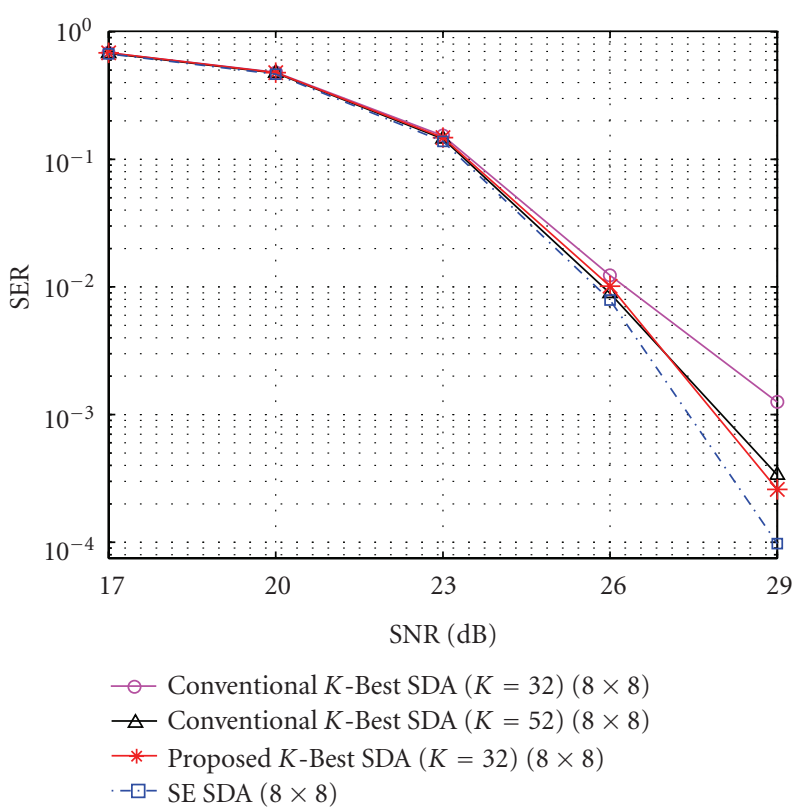

(a)

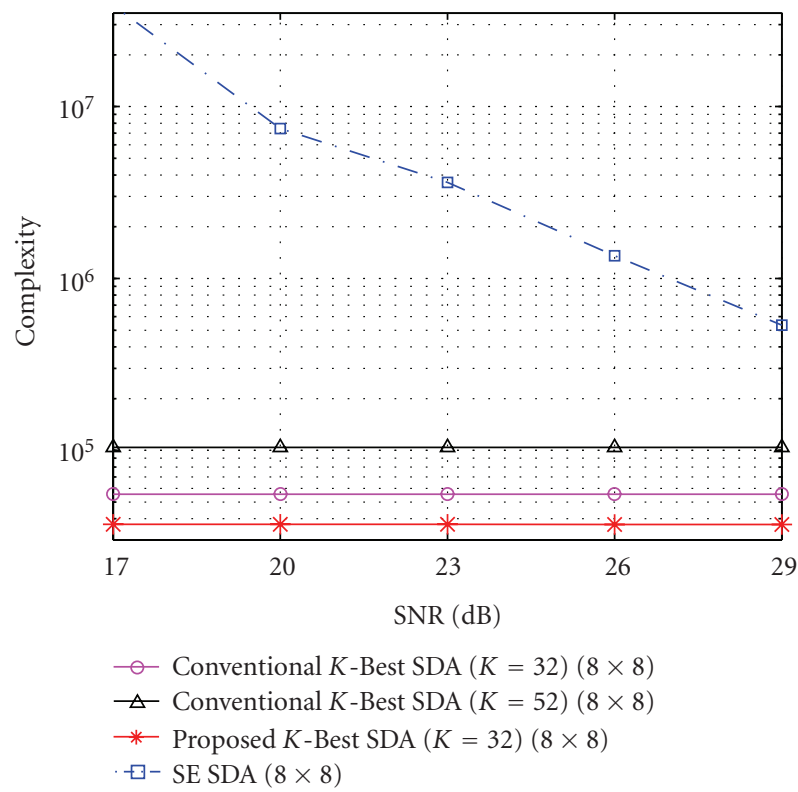

(b)

FIGURE 15: Performance and complexity of SDA for $8 \times 8$ MIMO systems with 16-QAM modulation. (a) SER. (b) Complexity. $K=32$ for proposed $K$-Best SDA; $K=32$ and 52 for conventional $K$-Best SDAs.

generation MIMO wireless communication systems such as IMT-Advanced.

\section{Appendix}

The expression in (13)-(15) shows that reordering the columns of $\mathbf{H}$ according to their vector norms in ascending order leads to

$$
\mathbf{H}_{o}=\left[\mathbf{h}_{o(1)}, \mathbf{h}_{o(2)}, \ldots, \mathbf{h}_{o(N)}\right]
$$

where $\left\|\mathbf{h}_{o(1)}\right\| \leq\left\|\mathbf{h}_{o(2)}\right\| \leq \cdots \leq\left\|\mathbf{h}_{o(N)}\right\|$. From [13], $\mathbf{h}_{o(i)}$ can be expressed as

$$
\mathbf{h}_{o(i)}=\sqrt{X_{i}} \boldsymbol{\theta}_{i}
$$

where $X_{i}$ is the $i$ th order statistic of $N$ independent $\operatorname{Gamma}(M, 1)$ distributed random variables with $X_{1} \leq X_{2} \leq$ $\cdots \leq X_{N}$ and $\left\{\boldsymbol{\theta}_{i}\right\} s$ are i.i.d. uniformly distributed on the unit sphere in $\mathbb{C}^{M}$. Note that $X_{i}$ and $\boldsymbol{\theta}_{i}$ are independent. With the $\mathrm{QR}$ decomposition of $\mathbf{H}_{o}=\mathbf{Q}_{o} \mathbf{R}_{o}$, we are now going to characterize the distribution of the square of the diagonal entries of $\mathbf{R}_{o}$ denoted by $r_{o, i, i}^{2}$.

Letting $\mathbf{Q}_{o}=\left[\mathbf{q}_{o(1)}, \mathbf{q}_{o(2)}, \ldots, \mathbf{q}_{o(N)}\right]$ and performing the QR decomposition of $\mathbf{H}_{o}$, we obtain

$$
\begin{aligned}
r_{o, i, i}^{2} & =X_{i}\left[1-\sum_{k=1}^{i-1}\left(\mathbf{q}_{o(k)}^{H} \boldsymbol{\theta}_{i}\right)^{2}\right]=X_{i}\left[1-\sum_{k=1}^{i-1} \boldsymbol{\theta}_{i}^{2}(k)\right] \\
& = \begin{cases}X_{i}, & \text { for } i=1 \\
X_{i} S_{i}, & \text { for } 2 \leq i \leq N,\end{cases}
\end{aligned}
$$

where

$$
S_{\mathrm{i}}=\left[1-\sum_{k=1}^{i-1} \boldsymbol{\theta}_{i}^{2}(k)\right],
$$

and $\boldsymbol{\theta}_{i}(\mathrm{k})$ denotes the $k$ th element of $\boldsymbol{\theta}_{i}$. Note that the second equation holds due to the fact that the distribution of $\boldsymbol{\theta}_{i}$ is invariant under the orthogonal transformation $\mathbf{Q}_{o}$. To derive the cdf of $r_{o, i, i}^{2}$, we should first obtain the probability density function (pdf) of $X_{i}$ and $S_{i}$. The pdf of $X_{i}$ is available in [39] as

$$
f_{X_{i}}(x)=\frac{N ![F(x)]^{i-1}[1-F(x)]^{N-i} f(x)}{(i-1) !(N-i) !},
$$

where

$$
\begin{gathered}
f(x)=\frac{x^{M-1} \cdot e^{-x}}{\Gamma(M)} \text { for } x>0, \\
F(x)=1-\sum_{i=0}^{M-1} \frac{x^{i}}{i !} e^{-x} .
\end{gathered}
$$

From [40], $\boldsymbol{\theta}_{i}$ can be modeled from a $2 M$-dimensional random vector $\mathbf{V}=\left[\begin{array}{llll}v_{1} & v_{2} & \cdots & v_{2 M}\end{array}\right]^{T}$ with $v_{i} \sim$ i.i.d. $N(0,1)$, where

$$
\boldsymbol{\theta}_{i}(k)=\frac{v_{2 k-1}+j \cdot v_{2 k}}{\|\mathbf{V}\|}=\frac{v_{2 k-1}+j \cdot v_{2 k}}{\sqrt{v_{1}^{2}+v_{2}^{2}+\cdots+v_{2 M}^{2}}},
$$

due to the fact that $\boldsymbol{\theta}_{i}^{H} \boldsymbol{\theta}_{i}=1, S_{i}$ can be rewritten as

$$
S_{i}=\left[1-\sum_{k=1}^{i-1} \boldsymbol{\theta}_{i}^{2}(k)\right]=\left[\sum_{k=1}^{M-i+1} \boldsymbol{\theta}_{i}^{2}(k)\right],
$$


substituting (A.7) into (A.8), we have

$$
S_{i}=\sum_{k=1}^{M-i+1} \boldsymbol{\theta}_{i}^{2}(k)=\frac{v_{1}^{2}+v_{2}^{2}+\cdots+v_{2 \cdot(M-i+1)}^{2}}{v_{1}^{2}+v_{2}^{2}+\cdots+v_{2 M}^{2}}=\frac{Q_{i}}{P_{i}},
$$

where $Q_{i}$ and $P_{i}$ are chi-square random variables with $2 \cdot(M-$ $i+1$ ) and $2 M$ degrees of freedom, respectively.

The joint pdf of $Q_{i}$ and $P_{i}$ is

$$
\begin{aligned}
f_{Q_{i}, P_{i}}(q, p) & =f_{Q_{i}}(q) \cdot f_{P_{i}-Q_{i}}(p-q) \\
& =f_{\chi(2 \cdot(M-i+1))}(q) \cdot f_{\chi(2 i-2)}(p-q) \\
& =\frac{q^{(M-i)} \cdot(p-q)^{(i-2)} \cdot e^{-p / 2}}{2^{M} \cdot \Gamma(M-i+1) \cdot \Gamma(i-1)}, \\
& \quad \text { for } p>0, q>0,
\end{aligned}
$$

where $f_{\chi(k)}(x)$ denotes the pdf of the chi-square random variable with $k$ degrees of freedom. The pdf of $S_{i}$ can be obtained by

$$
\begin{aligned}
f_{S_{i}}(s) & =\int_{-\infty}^{\infty}|p| f_{Q_{i}, P_{i}}(p s, p) d p \\
& =\int_{0}^{\infty} p \cdot \frac{(p s)^{(M-i)} \cdot(p-p s)^{(i-2)} \cdot e^{-p / 2}}{2^{M} \cdot \Gamma(M-i+1) \cdot \Gamma(i-1)} d p \\
& =\frac{s^{(M-i)} \cdot(1-s)^{(i-2)}}{2^{M} \cdot \Gamma(M-i+1) \cdot \Gamma(i-1)} \int_{0}^{\infty} p^{(M-1)} \cdot e^{-p / 2} d p \\
& =\frac{s^{(M-i)} \cdot(1-s)^{(i-2)} \cdot(M-1) !}{\Gamma(M-i+1) \cdot \Gamma(i-1)}
\end{aligned}
$$

Since $X_{i}$ and $S_{i}$ are independent, the joint pdf of $X_{i}$ and $S_{i}$ is

$$
f_{X_{i}, S_{i}}(x, s)=f_{X_{i}}(x) \cdot f_{S_{i}}(s) .
$$

The cdf of $r_{o, i, i}^{2}$ for $2 \leq i \leq N$ can be obtained by

$$
\begin{aligned}
F_{r_{o, i, i}^{2}}(r) & =\int_{0}^{1}\left\{\int_{0}^{r / s} f_{X_{i}, S_{i}}(x, s) d x\right\} d s \\
& =\int_{0}^{1} \int_{0}^{r / s} f_{X_{i}}(x) f_{S_{i}}(s) d x d s .
\end{aligned}
$$

Finally, the cdf of $r_{o, i, i}^{2}$ is as follows:

for $i=1$

$$
\begin{aligned}
& F_{r_{o, i, i}^{2}}(r)=\int_{0}^{r} \frac{N !}{(N-1) !(M-1) !}\left[\sum_{k=0}^{M-1} \frac{x^{k}}{k !} e^{-x}\right]^{N-1} \\
& \cdot x^{M-1} e^{-x} d x
\end{aligned}
$$

for $2 \leq i \leq N$

$$
\begin{gathered}
F_{r_{o, i, i}^{2}}(r)=C_{i i} \int_{0}^{1} \int_{0}^{r / s}\left[1-\sum_{k=0}^{M-1} \frac{x^{k}}{k !} e^{-x}\right]^{i-1}\left[\sum_{k=0}^{M-1} \frac{x^{k}}{k !} e^{-x}\right]^{N-i} \\
\cdot x^{M-1} e^{-x}(s)^{M-i}(1-s)^{i-2} d x d s,
\end{gathered}
$$

where

$$
C_{i i}=\frac{N !}{(N-i) !(M-i) !(i-1) !(i-2) !} .
$$

\section{Acknowledgment}

This work was supported by the National Science Council of Taiwan under Contract no. NSC 97-2221-E-009-056-MY2.

\section{References}

[1] E. Telatar, "Capacity of multi-antenna Gaussian channels," AT\&T Bell Labs Internal Technical Memorandum, June 1995.

[2] G. J. Foschini and M. J. Gans, "On limits of wireless communications in a fading environment when using multiple antennas," Wireless Personal Communications, vol. 6, no. 3, pp. 311-335, 1998.

[3] S. M. Alamouti, "A simple transmit diversity technique for wireless communications," IEEE Journal on Selected Areas in Communications, vol. 16, no. 8, pp. 1451-1458, 1998.

[4] G. J. Foschini, "Layered space-time architecture for wireless communication in a fading environment when using multielement antennas," Bell Labs Technical Journal, vol. 1, no. 2, pp. 41-59, 1996.

[5] A. J. Goldsmith, "Variable-rate variable-power MQAM for fading channels," IEEE Transactions on Communications, vol. 45, no. 10, pp. 1218-1230, 1997.

[6] S. Catreux, V. Erceg, D. Gesbert, and R. W. Heath, "Adaptive modulation and MIMO coding for broadband wireless data networks," IEEE Communications Magazine, vol. 40, no. 6, pp. 108-115, 2002.

[7] G. J. Foschini, G. D. Golden, R. A. Valenzuela, and P. W. Wolniansky, "Simplified processing for high spectral efficiency wireless communication employing multi-element arrays," IEEE Journal on Selected Areas in Communications, vol. 17, no. 11, pp. 1841-1852, 1999.

[8] G. D. Golden, C. J. Foschini, R. A. Valenzuela, and P. W. Wolniansky, "Detection algorithm and initial laboratory results using V-BLAST space-time communication architecture," Electronics Letters, vol. 35, no. 1, pp. 14-16, 1999.

[9] U. Fincke and M. Pohst, "Improved methods for calculating vectors of short length in a lattice, including a complexity analysis," Mathematics of Computation, vol. 44, pp. 463-471, 1985.

[10] B. Hassibi and H. Vikalo, "On the sphere-decoding algorithm I. expected complexity," IEEE Transactions on Signal Processing, vol. 53 , no. 8, pp. 2806-2818, 2005.

[11] H. Vikalo and B. Hassibi, "On the sphere-decoding algorithm II. generalizations, second-order statistics, and applications to communications," IEEE Transactions on Signal Processing, vol. 53, no. 8, pp. 2819-2834, 2005.

[12] C. P. Schnorr and M. Euchner, "Lattice basis reduction: improved practical algorithms and solving subset sum problems," Mathematical Programming, vol. 66, no. 2, pp. 181-199, 1994.

[13] W. Zhao and G. B. Giannakis, "Reduced complexity closest point decoding algorithms for random lattices," IEEE Transactions on Wireless Communications, vol. 5, no. 1, pp. 101-111, 2006. 
[14] Z. Guo and P. Nilsson, "Algorithm and implementation of the K-best sphere decoding for MIMO detection," IEEE Journal on Selected Areas in Communications, vol. 24, no. 3, pp. 491-503, 2006.

[15] A. Burg, M. Borgmann, M. Wenk, M. Zellweger, W. Fichtner, and H. Bölcskei, "VLSI implementation of MIMO detection using the sphere decoding algorithm," IEEE Journal of SolidState Circuits, vol. 40, no. 7, pp. 1566-1576, 2005.

[16] X. Huang, C. Liang, and J. Ma, "System architecture and implementation of MIMO sphere decoders on FPGA," IEEE Transactions on Very Large Scale Integration (VLSI) Systems, vol. 16, no. 2, pp. 188-196, 2008.

[17] K. W. Wong, C. Y. Tsui, R. S. K. Cheng, and W. H. Mow, "A VLSI architecture of a K-best lattice decoding algorithm for MIMO channels," in Proceedings of the IEEE International Symposium on Circuits and Systems (ISCAS '02), vol. 3, pp. 273-276, May 2002.

[18] Y. H. Wu, Y. T. Liu, H. C. Chang, Y. C. Liao, and H. C. Chang, "Early-pruned K-best sphere decoding algorithm based on radius constraints," in Proceedings of the IEEE International Conference on Communications (ICC '08), pp. 4496-4500, May 2008.

[19] Q. Li and Z. Wang, "Reduced complexity K-Best sphere decoder design for MIMO systems," Circuits, Systems, and Signal Processing, vol. 27, no. 4, pp. 491-505, 2008.

[20] M. Shabany and P. G. Gulak, "A $0.13 \mu \mathrm{m}$ CMOS 655Mb/s 44 64-QAM K-Best MIMO detector," in Proceedings of the IEEE International Solid-State Circuits Conference (ISSCC '09), pp. 256-257, February 2009.

[21] B. Shim and I. Kang, "Sphere decoding with a probabilistic tree pruning," IEEE Transactions on Signal Processing, vol. 56, no. 10, pp. 4867-4878, 2008.

[22] L. Qingwei and W. Zhongfeng, "Improved K-Best sphere decoding algorithms for MIMO systems," in Proceedings of the IEEE International Symposium on Circuits and Systems (ISCAS '06), pp. 1159-1162, May 2006.

[23] W. C. Jun, S. Byonghyo, A. C. Singer, and IK. C. Nam, "A low-complexity near-ml decoding technique via reduced dimension list stack algorithm," in Proceedings of the 5th IEEE Sensor Array and Multichannel Signal Processing Workshop (SAM '08), pp. 41-44, July 2008.

[24] S. Roger, A. Gonzalez, V. Almenar, and A. Vidal, "Combined K-best sphere decoder based on the channel matrix condition number," in Proceedings of the 3rd International Symposium on Communications, Control, and Signal Processing (ISCCSP '08), pp. 1058-1061, March 2008.

[25] S. Roger, A. Gonzalez, V. Almenar, and A. M. Vidal, "MIMO channel matrix condition number estimation and threshold selection for combined K-best sphere decoders," IEICE Transactions on Communications, vol. E92-B, no. 4, pp. 1380-1383, 2009.

[26] L. Azzam and E. Ayanoglu, "Reduced complexity sphere decoding for square QAM via a new lattice representation," in Proceedings of the 50th Annual IEEE Global Telecommunications Conference (GLOBECOM '07), pp. 4242-4246, November 2007.

[27] K. Amiri, C. Dick, R. Rao, and J. R. Cavallaro, "Novel sort-free detector with modified real-valued decomposition (M-RVD) ordering in MIMO systems," in Proceedings of the 50th Annual IEEE Global Telecommunications Conference (GLOBECOM '08), pp. 4217-4221, December 2008.
[28] M. Myllylä, M. Juntti, and J. R. Cavallaro, "Implementation aspects of list sphere detector algorithms," in Proceedings of the 50th Annual IEEE Global Telecommunications Conference (GLOBECOM '07), pp. 3915-3920, November 2007.

[29] M. Myllylä, M. Juntti, and J. R. Cavallaro, "Implementation aspects of list sphere decoder algorithms for MIMO-OFDM systems," Signal Processing, vol. 90, no. 10, pp. 2863-2876, 2010.

[30] H. L. Lin, R. C. Chang, and H. L. Chen, "A high-speed SDMMIMO decoder using efficient candidate searching for wireless communication," IEEE Transactions on Circuits and Systems II, vol. 55, no. 3, pp. 289-293, 2008.

[31] S. Mondal, K. N. Salama, and W. H. Ali, "A novel approach for K-best MIMO detection and its VLSI implementation," in Proceedings of the IEEE International Symposium on Circuits and Systems (ISCAS '08), pp. 936-939, May 2008.

[32] Z. Wen, "Multiway merging in parallel," IEEE Transactions on Parallel and Distributed Systems, vol. 7, no. 1, pp. 11-17, 1996.

[33] S. Mondal, A. M. Eltawil, and K. N. Salama, "Architectural optimizations for low-power K-best MIMO decoders," IEEE Transactions on Vehicular Technology, vol. 58, no. 7, pp. 31453153, 2009.

[34] S. Mondal, A. Eltawil, C. A. Shen, and K. N. Salama, "Design and Implementation of a sort-free K-best sphere decoder," IEEE Transactions on Very Large Scale Integration (VLSI) Systems, vol. 18, no. 10, pp. 1497-1501, 2009.

[35] K. Lee and J. Chun, "ML symbol detection based on the shortest path algorithm for MIMO systems," IEEE Transactions on Signal Processing, vol. 55, no. 11, pp. 5477-5484, 2007.

[36] A. K. Lenstra, H. W. Lenstra, and L. Lovász, "Factoring polynomials with rational coefficients," Mathematische Annalen, vol. 261, no. 4, pp. 515-534, 1982.

[37] H. C. Chang, Y. C. Liao, and H. C. Chang, "Low-complexity prediction techniques of K-best sphere decoding for MIMO systems," in Proceedings of the IEEE Workshop on Signal Processing Systems (SiPS '07), pp. 45-49, October 2007.

[38] A. Wiesel, X. Mestre, A. Pages, and J. R. Fonollosa, "Efficient implementation of sphere demodulation," in Proceedings of the IEEE Workshop on Signal Processing Advances in Wireless Communications (SPAWC '03), pp. 36-40, June 2003.

[39] N. Balakrishnan and A. C. Cohen, Order Statistics and Inference Estimation Methods, Academic Press, New York, NY, USA, 1991.

[40] M. E. Muller, "A note on a method for generating points uniformly on n-dimensional spheres," Communications of the ACM, vol. 2, pp. 19-20, 1959. 